Portland State University

PDXScholar

5-5-1993

\title{
Mit Lachen die Wahrheit zu sagen Entwicklungsgeschichte und Autorintention des Barockromans Simplicissimus
}

Ralf Genske

Portland State University

Follow this and additional works at: https://pdxscholar.library.pdx.edu/open_access_etds

Part of the German Language and Literature Commons Let us know how access to this document benefits you.

Recommended Citation

Genske, Ralf, "Mit Lachen die Wahrheit zu sagen Entwicklungsgeschichte und Autorintention des Barockromans Simplicissimus" (1993). Dissertations and Theses. Paper 4611.

https://doi.org/10.15760/etd.6495

This Thesis is brought to you for free and open access. It has been accepted for inclusion in Dissertations and Theses by an authorized administrator of PDXScholar. Please contact us if we can make this document more accessible: pdxscholar@pdx.edu. 
AN ABSTRACT OF THE THESIS OF Ralf Genske for the Master of Arts in German, presented May 5th, 1993.

Title: "Mit Lachen die Wahrheit zu sagen"

Entwicklungsgeschichte und Autorintention des

Barockromans Simplicissimus

APPROVED BY THE MEMBERS OF THE THESIS COMMITTEE:

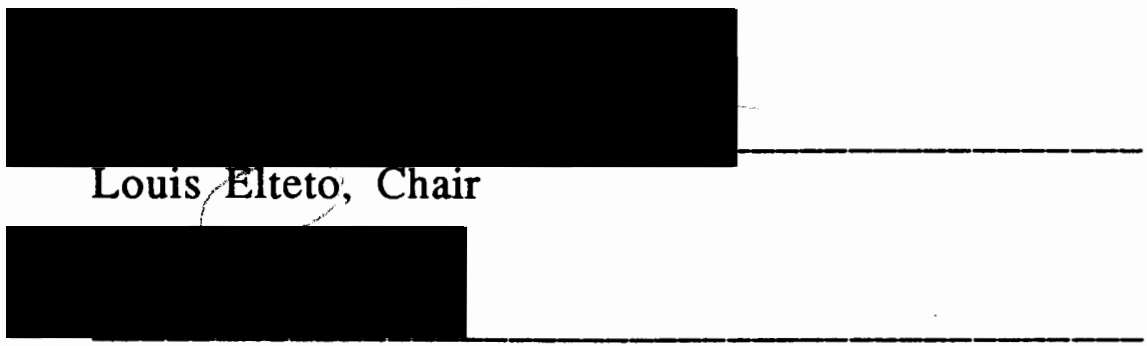

Linda Parshall

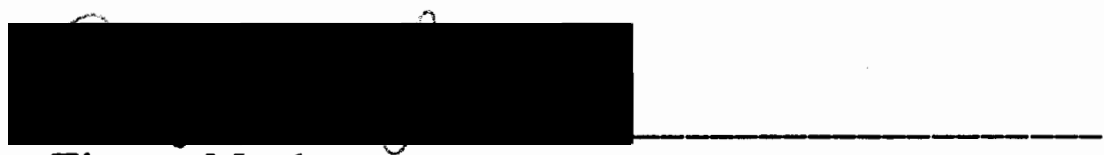

Timm Menke

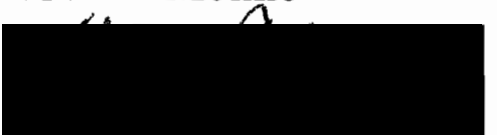

Steven Fuller

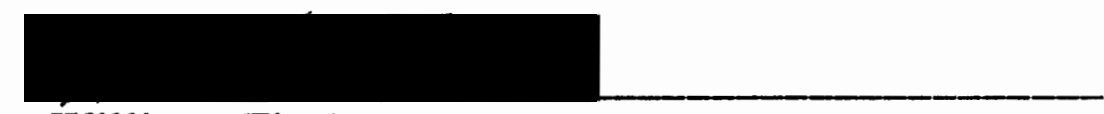

William Fischer

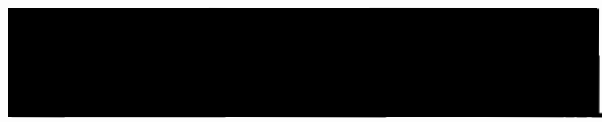

Franklin West 
The bloody epoch of the Thirty Years' War and the wide spread decline of German culture and social life is the major topic in Simplicissimus, the first High German novel written by Hans Jacob Christoph von Grimmelshausen in 1668. The purpose of this thesis is to summarize the development of this novel and to find Grimmelshausen's intention in writing his book which was immensely popular upon its publication, but disappeared into obscurity during the German classical period.

Since its rediscovery in the middle of the 19 th century, scholars have puzzled over the satirical meaning of Simplicissimus as well as the identity of its author. By concentrating on the sources available to Grimmelshausen, researchers have proposed a variety of interpretations of the text. Some see the book as Germany's best example of the Spanish picaresque novel, others include it in the genre of "Bildungsroman", whose classic example is Goethe's Wilhelm Meister. Many readers marvel over the satirical and witty language of the novel, while others admire its sophisticated structures.

It is evident that scholars have yet to reach a consensus on a comprehensive interpretation of Simplicissimus. Valuable insight could be gained from discovering Grimmelshausen's intentions. By searching the text carefully for statements made by the author about his opinion on social customs, war, family, religion etc., a composite picture of Grimmelshausen's world view was constructed.

By combining these assumed attitudes and opinions with his personal development and the social and historical influences of his 
time, the conclusion emerged that the author wrote his novel out of an ethical didactic urge to confront his readers with the ills of their time.

The economic, political and religious changes of the 17 th century uprooted the established medieval structures of European life, causing millions of deaths and devastation in large parts of Germany. Grimmelshausen saw the deterioration of life directly related to the changes in the beliefs and behaviors of his contemporaries and felt compelled to alarm his fellow men. A second motivational force for his literary endeavors was the necessity to supplement his small income as tavern owner, although he certainly could not have anticipated the astonishing success of his book.

The confusing array of literary forms in Simplicissimus is the result of the author's concern with his message which subordinated the adherence to a specific genre, but nevertheless produced a masterpiece of German literature. 


\title{
"MIT LACHEN DIE WAHRHEIT ZU SAGEN" ENTWICKLUNGSGESCHICHTE UND AUTORINTENTION DES BAROCKROMANS SIMPLICISSIMUS
}

\author{
von \\ RALF GENSKE
}

\begin{abstract}
A thesis submitted in partial fulfillment of the requirements for the degree of
\end{abstract}

\author{
MASTER OF ARTS \\ in \\ GERMAN
}

Portland State University

1993 


\section{TO THE OFFICE OF GRADUATE STUDIES:}

The members of the Committee approve the thesis of Ralf Genske presented on May 5th, 1993:

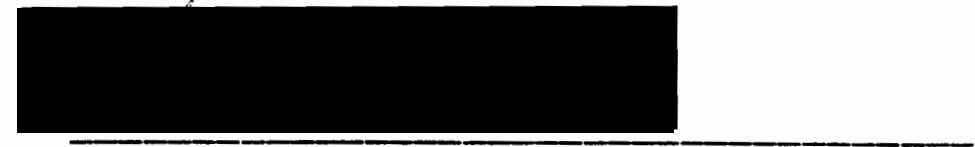

Louis Elteto, Chair

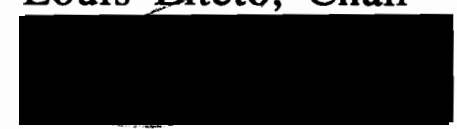

Linda Parshall

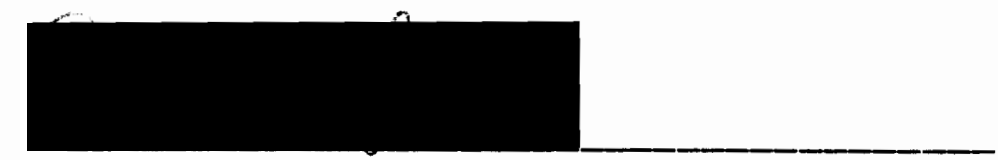

Timm Menke

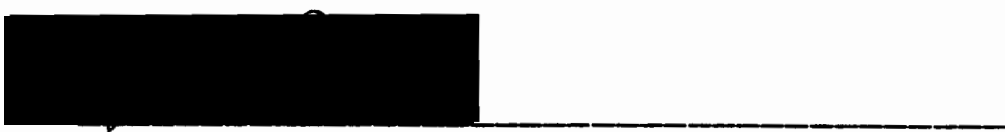

Steven Fuller

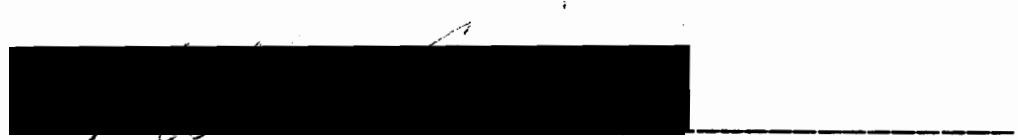

William Fischer

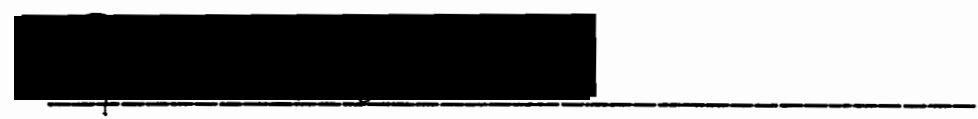

Franklin West

APPROVED:

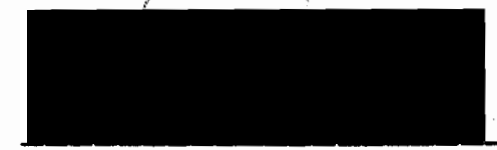

Linda Parshall, Chair, Department of Foreign Language and Literature

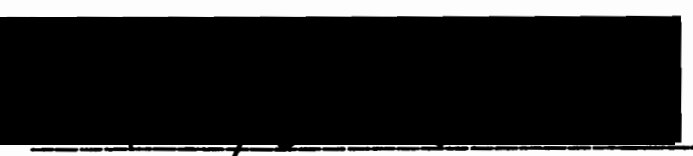

Roy W. Koch, Vice Provost for Graduate Studies and Research 


\section{ACKNOWLEDGMENTS}

Zwar ist die Forschung an einer These zumeist ein einsames Unterfangen, sie geschieht aber nicht in einem Vakuum. Auch diese Arbeit hätte ohne die tatkräftige Hilfe meiner Lehrer und Freunde nicht so entstehen können.

An dieser Stelle möchte ich mich daher bei allen Professoren der Deutschsprachenabteilung an der Portland State Universität bedanken, die mich während meines Studiums und der anschliessenden Assistentenzeit so wohlwollend unterstützt haben. Ganz besonders fühle ich mich Dr. Timm Menke verpflichtet, ohne den ich gar nicht auf den Gedanken gekommen wäre, als Graduate Student mein Studium fortzusetzen, und der mir auch weiterhin unzählige hilfreiche Hinweise gegeben hat. Ferner muß ich mich bei Dr. William Fischer für die wertvollen Anweisungen beim Sprachunterricht bedanken. Dank seiner pädagogischen Lehrstrategien werde ich ein besserer Deutschlehrer sein. Natürlich kann ich meinen Berater, Dr. Louis Elteto, nicht vergessen, durch den mein Interesse an der deutschen Literatur erst geweckt wurde. Er führte mich behutsam in die Schönheit der deutschen Klassik ein, und setzte mich auf die Spur Hans Jacob Christoph von Grimmelshausens, den kennenzulernen mir viel Vergnügen gebracht hat. Ich bedanke mich herzlich. 
INHALTSANGABE

SEITE

ACKNOWLEDGMENTS. ................... iii

\section{KAPITEL}

I EINLEITUNG $\ldots \ldots \ldots \ldots \ldots \ldots \ldots \ldots \ldots \ldots \ldots$

II GESCHICHTLICHE UND SOZIALE ASPEKTE DES

17. JAHRHUNDERTS . . . . . . . . . . . . 4

III DAS LEBEN GRIMMELSHAUSENS $\ldots \ldots \ldots \ldots \ldots \ldots$

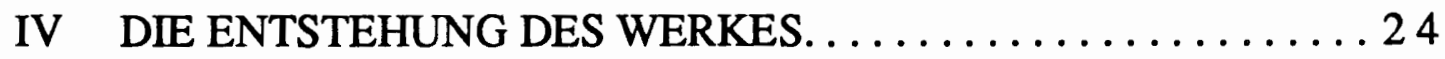

V DIE INTERPRETATION DES ROMANS DURCH DIE

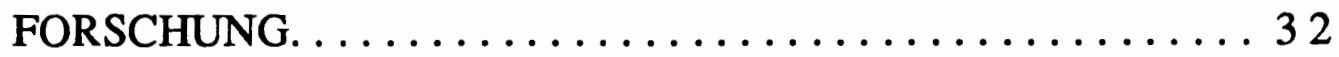

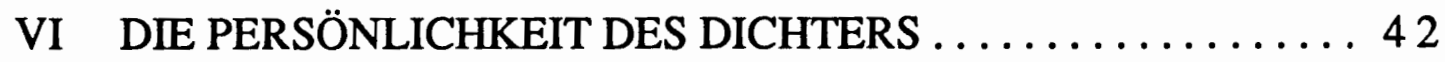

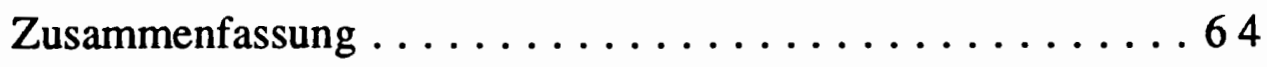

VII GRIMMELSHAUSENS ABSICHT BEI DER SCHAFFUNG SEINES HAUPTWERKES $\ldots \ldots \ldots \ldots \ldots \ldots \ldots 6$

QUELLENNACHWEIS . .................. 77 


\section{KAPITEL}

\section{EINLEITUNG}

Als der Schultheiß des kleinen Schwarzwaldortes Renchen 1668 sein schweres, vielseitiges Manuskript dem Verleger Felßecker in Nürnberg überreichte, gab er zwar den ersten neuhochdeutschen Roman zum Druck frei, ${ }^{1}$ führte gleichzeitig aber ein Werk in die deutsche Literatur ein, dessen Herkunft, Bedeutung, Wirkung und Beurteilung umstritten und geheimnisvoll wie kaum ein anderes ist.

Schon nach dem weit ausladenden Titel: Der Abentheuerliche Simplicissimus Teutsch. Das ist die Beschreibung des Lebens eines seltsamen Vaganten genannt Melchior Sternfels von Fuchshaim. wo und welcher gestalt er nemlich in diese Welt kommen, was er er solche wieder freiwillig quittierte. Überaus lustig und männiglich nutzlich zu lesen, folgte mit dem Namen des Autoren das erste Rätsel des Buches. Wer verbarg sich hinter dem ungewöhnlichen Namen "German Schleifheim von Sulsfort"?

Es dauerte immerhin bis 1837 bevor das Geheimnis des Autorenanagramms dank der scharfsinnigen Arbeiten von Kurz,

${ }^{1}$ Kurt Böttcher, Lexikon deutschsprachiger Schriftsteller (Leipzig: Bibliographisches Institut, 1987), 190 
Echtermeyer und Passow gelüftet, und das Buch als das Produkt des Hans Jakob Christoph von Grimmelshausen entlarvt wurde. Geklärt aber wurde die Frage nicht, warum der geheimnisvolle Schriftsteller seine Identität unter einem Pseudonym versteckte.

Obwohl seit der Mitte des 19. Jahrhunderts, und besonders zu Beginn des 20. Jahrhunderts, eine Vielzahl von Büchern, gewichtigen Artikeln und Dissertationen sich mit dem Leben und Werk des schreibenden Bürgermeisters befaßt haben, blieben viele Fragen unbeantwortet und beschäftigen auch heute noch die Literaturforschung.

Schon die elementare Frage nach der Gattungsbestimmung des Simplicissimus stößt auf ein Chaos von Meinungen. Die einen halten Grimmelshausen für einen unheilbaren Schwätzer und Fabulierer, ${ }^{2}$ der mit seiner Geschichte nur ein Dokument seiner unbändigen Erzähllust schaffen wollte. Andere sind begeistert über den stufenweisen Aufbau und die systematische Anlage des Stoffes und zählen daher den Autoren zu den Großen der deutschen Literatur, 3 während die Dritten im Simplicissimus einen barocken Entwicklungsroman sehen, der sich zwischen Wolframs Parzifal und Goethes Wilhelm Meister einordnen läßt.

Wohl sind sich die meisten Stimmen über die Wertschätzung

${ }^{2}$ E. Cohn, Gesellschaftsideal und Gesellschaftsroman des 17. Jahrhunderts (Berlin: Emil Ebering Verlag, 1921), 13

3J. Alt, "Selbstbiografie und Typenaufbau," in G. Weydt, Der Simplicissimusdichter und sein Werk (Darmstadt: Wissenschaftliche Buchgesellschaft, 1969), 200 
des Dichters einig, aber mit der Flut der Veröffentlichungen weitete sich auch der Strom der Interpretationen. In dieser Arbeit soll nun der Versuch unternommen werden, die Entwicklungsgeschichte des Simplicissimus nachzuvollziehen und ihn durch die Persönlichkeit seines Dichters im Zusammenhang seiner Zeit zu erklären. Dabei soll besonders die Frage nach dem möglichen Grund für die Entstehung des Buches im Vordergrund stehen. Durch Beantwortung der Frage: Warum mag Grimmelshausen seinen Roman geschrieben haben? soll einer allgemeinen Typologie des Simplicissimus Hilfestellung geleistet werden, die die Interpretation des Buches sehr erleichtern würde. 


\section{KAPITEL}

\section{GESCHICHTLICHE UND SOZIALE ASPEKTE \\ DES 17. JAHRHUNDERTS}

Um dem Inhalt des Simplicissimus, aber auch um der Person seines Autoren näher zu kommen, dürfen die geschichtlichen und sozialen Strömungen jener Zeit nicht außer Acht gelassen werden. Die politische und geistige Situation des 16. und 17. Jahrhunderts mußte gerade auf einen so interessierten und aufgeweckten Beobachter wie Grimmelshausen erheblichen Einfluß haben. Alle seine Schriften zeugen von der Aufnahme und aktiven Verarbeitung der oftmals widersprüchlichen Verhältnisse, die die Welt des Schwarzwälder Schaffners und Bürgermeisters ausfüllten.

Was er und seine ähnlich aufgeschloßenen Zeitgenossen sahen, war eine Periode tiefgreifender Veränderungen einer Gesellschaft im Umbruch, eine Epoche der blutigsten Konflikte und des größtmöglichen menschlichen Leidens. Das 17. Jahrhundert ist das kriegerischste Jahrhundert der bisherigen menschlichen Geschichte. Bis auf sieben $(1610,1669-71,1680-82)$ gab es keine Jahre zwischen 1600 und 1699, in denen kein Krieg zwischen europäischen Mächten 
stattfand. 1 Stand zu Beginn der Epoche das Leben noch auf relativ festen spätmittelalterlichen Feudalgrundlagen, so war es zum Ende des 17. Jahrhunderts in eine ungewisse Neuzeit katapultiert worden. Diese Entwicklung begann allerdings nicht erst mit dem Ausbruch des Dreißigjährigen Krieges, sondern sie zeichnete sich bereits fast hundert Jahre vorher am Horizont ab.

Die Religionsfrage schwelte seit dem Reichstag zu Worms 1520 trotz des Religionsfriedens von Augsburg im Jahre 1555 auch weiterhin. Der komplizierte Aufbau der mittelalterlichen Stände begann sich im Laufe des 16. Jahrhunderts unter dem Druck der aufstrebenden Territorialfürsten aufzulösen. Gleichzeitig fand eine Vermögensverlagerung statt, bei der das alte Stadtpatriziat, die Zunftmeister, Kaufherren und Bankiers, die neuen Führungskräfte der frühabsolutistischen Hofgesellschaften neben sich anerkennen mußten. Der alt-angestammte Adel verlor durch den Vormarsch handeltreibender Bürger an Ansehen und Stellung. Adelstitel und Schlösser wurden käufliche Gebrauchsgegenstände, wie das Beispiel des Dr. Küffer auf der Ullenburg in Grimmelshausens direkter Umgebung veranschaulicht.

Hand in Hand mit dieser Verweltlichung des täglichen Lebens ging ein geistiger Konflikt zwischen jenseitsbezogenem Vergänglichkeitsdenken und dem Wunsch nach weltlicher Ordnung, der durch die Kriegsverwüstungen besonders an Aktualität gewann. Diese

${ }^{1}$ George Clark, The Seventeenth Century (Oxford: University Press, 1969), 98 
vielfältigen Entwicklungen bewegten die Menschen des 17. Jahrhunderts und schlugen sich mehr oder weniger deutlich in den geistigen Manifestationen dieser Zeit nieder.

Der Bauernkrieg in Süddeutschland, der mit der blutigen Niederlage der Landarbeiter 1525 endete, war das erste Anzeichen einer sich auflösenden Feudaltradition. In der Folgezeit erwies sich dabei Frankreich als führende Kraft, da sich dort politisch orientierte Schriftsteller und Staatsrechtler dem Problem der Staatsgefährdung durch Bürgerkriege und Revolutionen annahmen. Jean Bodin, der philosophische Wegbereiter des Absolutismus, entwickelte 1577 die Neudefinition des Staates als "die höchste, von Gesetzen ledige Gewalt über Bürger und Untertanen".2 Andere europäische Denker, wie Justus Lipsius oder Thomas Hobbes, rechtfertigten die Souveränmacht des Staates durch Berufung auf das göttliche Recht und das Naturrecht, die beide als Sicherheit vor gottloser Anarchie bedingungslose Unterwerfung des Individuums verlangten.

Mit dieser steigenden Rolle des Staates ging ein stetiges Absinken der traditionellen Stände einher. Die Landesfürsten und der Hochadel bestimmten zunehmend das politische und gesellschaftliche Bild des Reiches. In den Städten, die als Machtzentren parallel zu den Ständen an Einfluß verloren, bildeten sich scharf nach Vermögen getrennte Bürgerschichten heraus. Neben hohem Klerus und adeligen Offizieren stiegen hohe Beamte und Universitätsabsolven-

2 V. Meid, Grimmelshausen, Epoche-Werk-Wirkung (München: Verlag C. H. Beck, 1984), 13 
ten im Ansehen auf, während die breite, "ungültige" Masse sich aus Handwerkern, Krämern, Tagelöhnern und Dienstboten zusammensetzte. ${ }^{3}$ Als Unterstand galten Abdecker, Scharfrichter und Bettler.

Die Situation der Bauern war von ihrer geographischen Lage abhängig. Im süddeutschen Raum gab es noch freie Reichsdörfer, aber in den meisten anderen Gebieten verloren die Bauern im Laufe des 16. und 17. Jahrhunderts zusehends an rechtlicher Gewalt und Ansehen.

Mit dem Ausbruch des 30jährigen Krieges begann eine krasse Verschlechterung des bäuerlichen Lebens. Weiträumige Verwüstungen der Agrarlandschaft und Bevölkerungsverluste von rund $60 \%$ stellten die Landwirtschaft in den besonders vom Krieg betroffenen Gebieten (Rheinland-Pfalz, Hessen, Württemberg, Thüringen, Schlesien, Mecklenburg und Ost-Pommern) vor ernste Überlebensfragen. ${ }^{4}$ Die unbeschützten Bauernsiedlungen waren der Willkür der Soldateska hilflos ausgeliefert, was diese mit ungeheuerer Grausamkeit ausnutzte. Grimmelshausen lieferte ein höchst lebensnahes Bild der verzweifelten Lage der Bauern mit seiner Beschreibung der Zerstörung des Hofes seines Knan. ${ }^{5}$ Man kann dabei sicher sein, daß er bei dem Bericht von selbsterlebten

${ }^{3}$ H. D. Gebauer, Grimmelshausens Bauerndarstellung: Literarische Sozialkritik und ihr Publikum (Marburg: N. Elwert Verlag, 1977), 413

${ }^{4}$ Hajo Holborn, History of Modern Germany 1648-1840 (New York: Alfred Knopf, Publ., 1973), 22

${ }^{5} \mathrm{H}$. J. C. v. Grimmelshausen, Der abenteuerliche Simplicissimus (Darmstadt: Wissenschaftliche Buchgesellschaft, 1985), Buch I, Kapitel 4 
Szenen ausgegangen ist. 6

Das Opfertum und die Hilfslosigkeit der Landbevölkerung veranschaulicht deutlich die Veränderungen, die im Verhältnis zwischen den Bauern und den Feudalherren im Laufe des 16. Jahrhunderts eingetreten waren. Die Bauern standen auch weiterhin unter dem Druck der adeligen Landeigentümer, ständig höhere Abgaben leisten zu müssen, ohne deren Schutz geniessen zu können, wie es im feudalen Mittelalter der Fall war. Für keinen Stand waren die Entwicklungen des 16. und 17. Jahrhunderts so nachteilig, wie für den Bauernstand.7

Auch in der zeitgenössischen Literatur spiegelte sich das Leiden der Bauern wider. So waren die Leidtragenden in Justus Georg Schottelius' Friedens Sieg ebenfalls die Bauern, und auch im Friedensspiel Irenaromachia des holsteinischen Dichters Johannes Rist gehen die Bauern ihrem sicheren Untergang entgegen. 8

Mit dem Aufstieg der Territorialfürsten trat eine Zentralisierung der Macht ein, die sich um die Fürstenhöfe gruppierte. Die Verwaltung der Gebiete übernahm eine aus gebildeten Bürgern und Aristokraten geformte Beamtenschaft, die sich zunehmend nach dem französischen Modell orientierte. Zeremonien, Sprache und Umgangsformen imitierten den französischen Hof und beeinflußten die Einstellung und Gedankenwelt. Es ist bezeichnend, daß die erste Uni-

\author{
${ }^{6}$ H. D. Gebauer, 83 \\ ${ }^{7}$ ebda, 87 \\ ${ }^{8}$ ebda, 93
}


versitätsvorlesung in deutscher Sprache im Jahre 1688 durch Christian Thomasius an der Universität in Leipzig über das Thema "Welcher Gestalt man denen Franzosen im gemeinen Leben und Wandel nachahmen solle" gehalten wurde. 9 Französische Worte infiltrierten die deutsche Sprache, der im Gegensatz zum Französischen, Spanischen oder Italienischen ein unterstützendes kulturelles Zentrum fehite. 10

Obwohl es im Laufe des 16. Jahrhunderts eine große Anzahl reformatorischer und gegenreformatorischer Schriften in deutscher Sprache gegeben hatte, fehlte es an grammatischer und stilistischer Einheit. Latein war auch im 17. Jahrhundert noch die offizielle Sprache der Kirche und der intellektuellen Kreise Europas. Mit der Ablösung Lateins durch die französische Sprache drohte Deutsch als Kultur- und Schriftsprache drittklassig zu werden. Es bestand die Gefahr eines allmählichen Versiegens der deutschen Sprache als kulturelles Kommunikationsmittel, was Martin Opitz $1624 \mathrm{zu}$ seinem Buch von der Deutschen Poeterey Anlaß gab.

Die deutschen Dichter des 17. Jahrhunderts begannen mit der schweren Bürde, Deutsch nicht nur dem Lateinischen ebenbürtig

${ }^{9}$ Leonard Forster, "Neo-Latin Tradition and Vernacular Poetry," in Gerhard Hoffmeister, German Baroque Literature: The European Perspective (New York: Frederick Unger Publ., 1983), 94

${ }^{10} \mathrm{~W}$. Busch, Hans Jakob Christoffel von Grimmelshausen (Frankfurt: Verlag Moritz Diesterweg, 1968), 27 
zu gestalten, sondern auch gegen Französisch zu verteidigen.11

Für eine Zeit schrieben einige Dichter, wie Opitz oder Paul Fleming, zweisprachig, lateinisch und deutsch, wobei die Leserschaft ausschlaggebend war. Der angesehene Mathematiker und Philosoph Wilhelm von Leibniz veröffentlichte die Schrift Unvorgreiffliche Gedanken betreffend die Ausübung und Verbesserung der deutschen Sprache auf Deutsch, während er alle anderen seiner Arbeiten in lateinischer oder französischer Sprache abfaßte. Es war aber auch Leibniz, der 1667 dichtete:

Was lobt man viel die Griechen?

Sie müssen sich verkriechen, wenn sich die teutsche Musa regt.

Was sonst die Römer gaben, kann man zu Hause haben, nachdem sich Mars bei uns gelegt. ${ }^{12}$

Ein nicht unbeträchtlicher Stolz spricht aus diesen Zeilen, der wohl durch die Anwesenheit der oftmals ausländischen Soldaten während des langen Krieges hervorgerufen worden war.

Die Verheerungen des Krieges riefen eine existentielle Krise hervor, bei der sich die Bewältigung des Vergänglichkeitsbewußtseins allen Irdischen im Rahmen der ewigwährenden göttlichen Ordnung zu einem zentralen Thema emporstilisierte. Die Leiden der Menschen brachte eine Neubelebung der stoischen Weltanschauung, die als Kernpunkt die Anleitung zum tugendhaften Handeln anbot. Das

${ }^{11}$ Peter Schaeffer, "Baroque Philology: The Position of German in The European Family of Languages," in Hoffmeister, 73

${ }^{12} \mathrm{~L}$. Forster, 91 
Übel in der Welt diente dazu, die Tugend zu erproben und zu verstärken. Unglück und Kriegsleiden hatten ihre Berechtigung als göttliche Strafe, Prüfung und Bewährungsmöglichkeit. ${ }^{13}$ Aber die stoische Welteinstellung war doch nur eine Übergangsphase, die in ein neues System des absoluten Gesellschaftskonformismus überleitete.

Die Literatur reflektierte das aktuelle Zeitgeschehen. Ihr fiel dabei neben dem Erbauungsaspekt vor allem der Lehrzweck zu. Martin Opitz sah in seinem Buch von der Deutschen Poeterey den Zweck der Dichtung in "Vberredung vnd vnterricht auch ergetzung der Leute".14 Die Literatur stellte sich daher als "Gesellschaftsdichtung" in den Dienst des Staates, dem sie durch die Vermittlung ethischer, politischer und sozialer Normen gefügige Untertanen schaffte. Die Anerkennung und Förderung der großen Barockdichter Opitz, Lohenstein, Gryphius und Zesen, die als gelehrte Beamte im Dienst des Adels standen, wurde durch ihre Adelserhebung noch unterstrichen. Diese Anbiederung an die fürstlichen Höfe kostete den Barockschriftstellern allerdings auch ihre künstlerische Freiheit.

Auch Grimmelshausen, dessen Großvater das Adelsprädikat 'von' aus seinem Namen gestrichen hatte, nahm den Adelstitel während seines Lebens wieder an, was auf den Wunsch schließen

${ }^{13}$ Friedrich Gaede, Humanismus. Barock. Aufklärung (Bern \& München: Francke Verlag 1971), 119

${ }^{14} \mathrm{~V}$. Meid, 50 
läßt, sich den aristokratischen Kreisen anzunähern. Koschlig vermutet, da $B$ es dem Bürgermeister aus dem Renchtal um Anerkennung, Achtung, vielleicht sogar Gleichberechtigung gegangen war, wobei es aber keinerlei Belege dafür gibt, daß er sich jemals in einem echten literarischen Abhängigkeitsverhältnis befunden hat.15 Auf das gespannte Verhältnis des schreibenden Schultheiß mit den Sprachgesellschaften und deren propagierten Dichtersprache wird im 5. Kapitel noch näher eingegangen.

Als direkte Folge des Konfessionsstreites Luthers konnte man stark aufkommende Zweifel an der althergebrachten Glaubensvorstellung im 16. und besonders im 17. Jahrhundert ausmachen, die sich in einer veränderten gesellschaftlichen Einstellung der Kirche gegenüber niederschlugen. Nicht von ungefähr fiel der kirchliche Baueifer diesem veränderten Weltbild zum Opfer. Der lange Krieg zehrte zusätzlich an der Finanzkraft und dem Förderungswillen der geistlichen und weltlichen Bauherren, was in der Einstellung der Bauarbeiten an den kirchlichen Großprojekten, wie dem Kölner, Regensburger oder Ulmer Dom, sichtbar wurde.

Waren im 16. Jahrhundert die Konfessionen noch die treibende Kraft hinter den politischen, geistigen und sittlichen Entwicklungen gewesen, so verloren sie diese Fähigkeit im Laufe des 17. Jahrhunderts. Der Krieg unterhöhlte die Überzeugungskraft der Kirche ebenso wie der Siegeszug der empirischen Naturwissenschaften und der

15 Manfred Koschlig, Das Ingenium Grimmelshausens und >das Kollektiv< (München: Verlag C. H. Beck, 1977), 106 
rationalistischen Philosophie. Zwar rief dieser Wandel keine kurzfristigen spektakulären Veränderungen hervor, aber es begann sich doch die Tendenz abzuzeichnen, die Konfessionen als nebensächliche Notwendigkeit abzutun.16

Die wirtschaftliche Lage Deutschlands vor, während und nach dem 30jährigen Krieg war sehr orts- und standesabhängig. Für das 16. Jahrhunderts läßt sich allgemein von einer stetigen Expansion der Gesamtwirtschaft bei ständig zunehmender Inflation reden. ${ }^{17}$ Durch stagnierende und sogar rückläufige Produktion, besonders in der Silber- und Kupfergewinnung, kam es bei ständig ansteigenden Kosten zum Anfang des 17. Jahrhunderts zu schwerwiegenden Finanzkrisen und Bankrotterklärungen großer Handelshäuser (Welser 1614), aber auch die Staatshaushalte sahen sich unter Druck gesetzt.

Die Fürsten begannen das Münzrecht an Privatleute zu verpachten, was eine rasche Verschlechterung des Münzwertes nach sich zog. Besonders die lohnabhängigen Schichten der Bevölkerung litten unter den betrügerischen Machenschaften dieser Münzverfälscher, im Volksmund "Kipper und Wipper" genannt. Der Edelmetallgehalt der Münzen nahm so stark $a b$, daß diese "vfm Wasser schwimmen vnd leichtlich durch den Athem fortgetrieben werden können".18 In

${ }^{16}$ Bruno Gebhardt, Handbuch der deutschen Geschichte. Bd. 2 (Stuttgart: Union Verlag, Stuttgart, 1961), 199

17 V. Meid, 23

${ }^{18}$ Roland Franz Schmiedt, Deutsche Geschichte Bd. 1 (Berlin: Deutscher Verlag der Wissenschaften, 1967), 620 
vielen Städten des Reiches, so in Wittenberg, Leipzig und Jena, kam es dann 1624 zu gewalttätigen Aufständen, die die Fürsten dazu zwangen, das Geldwesen wieder selber zu kontrollieren. Immer deutlicher verschärfte sich in den Menschen das Bewußtsein von der Macht des Geldes.

Der Krieg brachte dann den Ruin der besetzten Gebiete, da die Heerführer, allen voran Wallenstein, dazu übergingen, ihre Soldaten durch Ausbeutung der besetzten Gebiete zu besolden. Dazu kam der Ausbruch der Pest, die die Bevölkerung zusätzlich stark dezimierte. Insgesamt hinterließ der 30jährige Krieg ein verwüstetes Land und eine ausgeblutete Bevölkerung.

Obwohl die meisten deutschen Territorialstaaten zu klein waren, um einen unabhängigen Wiederaufbau zu finanzieren, sahen sich doch alle Regierenden dazu gezwungen, ein neues Wirtschaftssystem zu errichten, das den veränderten sozialen Bedingungen entsprach und die Kriegsschäden beheben konnte. Um ihre eigene Macht zu festigen, kam es nach dem Krieg zu einer engen Zusammenarbeit der Staatsmächte mit der Wirtschaft, die weit über das feudalistische Abgaben- und Lehensprinzip hinausging, und die zu einer merkantilistischen Wirtschaftspolitik führte. ${ }^{19}$

Durch gezielte Schritte, wie Vereinheitlichung und Regelung des Zahlungswesens, der Zölle, der Maße und Gewichte, sowie durch Verbesserungen der Handelsbedingungen für den Inlandmarkt 
versuchten die absolutistisch regierten Staaten, allen voran Frankreich, ihren Außenhandel zu vermehren. Durch Förderung der Produktivität des einheimischen Gewerbes nahmen die $\mathrm{Re}$ gierungsinstitutionen verstärkten Anteil an der Wirtschaftskraft ihres Landes.

In den größeren Territorien des Reiches, namentlich in Österreich, Brandenburg, Bayern und Sachsen, kam es dann im späten 17. Jahrhundert durch zunehmende Zentralisierung und verstärkte Rationalisierungsmaßnahmen $\mathrm{zu}$ einem anhaltenden wirtschaftlichen Aufschwung, der durch Zuwanderungen bestimmter Volksgruppen (Hugenotten nach 1685) noch stärkere Impulse erhielt.

Aus den angeführten Schwerpunkten wird klar, daß das 17. Jahrhundert wahrhaftig eine Epoche des Umsturzes war, wie sie die deutschen Menschen bis zum 20. Jahrhundert nicht mehr erleben mußten. Auch in unserem Jahrhundert erfuhren die Deutschen gravierende Wechsel im politischen, sozialen und wirtschaftlichen Gefüge. Zwei lange Kriege verwüsteten die deutschen Lande und das Vertrauen der Menschen auf ein beständiges, friedliches $\mathrm{Zu}$ sammenleben im europäischen Raum.

Es ist kein Wunder, daß Geschichtswissenschaftler Parallele zwischen dem Jahrhundert des 30jährigen Krieges und dem des 1. und 2. Weltkrieges ziehen können. In beiden Zeitaltern bewiesen aber die betroffenen Menschen durch zähen Überlebenswillen und Öffnungsfähigkeit zu neuem Gedankengut, daß auch durch Chaos und Tumult eine Bereicherung der Kultur möglich ist. 
Das 17. Jahrhundert fand in Grimmelshausen einen hervorragenden Chronisten dieser Entwicklungen, da er wie sonst niemand seiner Zeit die psychologischen und sozialen Veränderungen mit feinfühliger Präzision aufnahm. In dieser Rolle dürfte letztendlich sein größter Wert liegen. 


\section{KAPITEL}

\section{DAS LEBEN GRIMMELSHAUSENS}

Für die Interpretation eines literarischen Werkes ist der Wert einer Autorenbiographie zweifelhaft und umstritten. Die angewandte Methodik der Literaturwissenschaft bestimmt zum größten Teil die Abhängigkeit eines Werkes von dem Leben seines Erstellers. Wie J. H. Scholte behauptet, ist die Lebensanschauung immer wichtiger als die Lebensgeschichte. 1 Trotzdem sind beide nicht eindeutig voneinander zu trennen, denn der Gang eines Lebens hat den größten Einfluß auf Meinungsbildung und Schaffenskraft des Menschen.

Im Fall des Simplicissimus-Dichters ging die Forschung lange Zeit von der Annahme aus, die Entwicklung des Titelhelden Simplicius mit der des Verfassers gleichsetzen zu können. Obwohl sich diese Annahme als leichtfertig und letztendlich unhaltbar erwies, behält der Lebenslauf des Simplicissimus doch seine Anziehungskraft für die Grimmelshausenforschung, da aus den wichtigen ersten zwei Jahrzehnten des Dichters, und das ist genau die Zeit, die er in seinem Roman detailiert beschrieb, sich keinerlei dokumentarisches Material hat auffinden lassen. So müssen die Lücken im Lebenslauf durch

${ }^{1}$ J. H. Scholte, Der Simplicissimus und sein Dichter (Tübingen: Max Niemeyer Verlag, 1950), 131 
sorgfältiges Vergleichen und vorsichtiges Abwägen der vermutlich autobiographisch gemachten Bemerkungen und Hinweise in seinen Geschichten geschloßen werden. Dabei wird neben seinen Kalendergeschichten, und da besonders der Ewigwährende Calender von 1671, hauptsächlich Grimmelshausens Hauptwerk Simplicissimus zu Rate gezogen.

Man ist sich heute einig darüber, daß Hans Jacob Christoph von Grimmelshausen 1621 oder 1622 im lutherischen Gelnhausen in Hessen, nordöstlich von Frankfurt, geboren wurde. Offenbar ist sein Vater Johann Christoph schon kurz nach seiner Geburt verstorben, denn die Mutter heiratete 1627 den Sohn eines Frankfurter Buchhändlers und verließ Gelnhausen. Hans Jacob blieb vermutlich in der Obhut seines Großvaters Melchior Christoph, der in Gelnhausen eine Bäckerei betrieb, zurück. Zu der formellen Schulausbildung gehörte wohl auch der Besuch der Lateinschule in Gelnhausen, die aber wahrscheinlich nur zu den Grundkenntnissen des Jungen beigetragen haben dürfte, ${ }^{2}$ da die Stadt im Anschluß an die Schlacht bei Nördlingen am 5. und 6. September 1634 von kaiserlichen Truppen geplündert und völlig zerstört wurde.

Durch diese einschneidende Begebenheit wurde der erst $12 \mathrm{jäh}$ rige Junge in den Malstrom des Dreißigjährigen Krieges gezogen. Über den Verlauf seiner folgenden vier Kriegsjahre lassen sich allerdings nur Vermutungen anstellen, die sich aber durch Ver-

2J. H. Scholte, 132 
gleiche der geschichtlichen Dokumente mit den Andeutungen Grimmelshausens in seinen Veröffentlichungen recht gut absichern lassen. Gustav Könnecke hat 1926 ein in mühsamster Kleinarbeit zusammengetragenes Buch herausgebracht, in dem er die Kriegsjahre des Simplicissimus-Autoren nachvollzieht. ${ }^{3}$ Diese Arbeit wird auch heute noch als ein Eckpfeiler der Grimmelshausenforschung anerkannt.

Die Bewohner Gelnhausens, und Hans Jacob weilte wohl unter ihnen, flüchteten sich nach der Zerstörung ihrer Stadt in die nahe Festung Hanau. Vermutlich wurde der Junge im Winter 1635 durch einen Trupp kroatischer Soldaten im Umfeld der Stadt aufgegriffen und entführt. ${ }^{4}$ Am 25. Februar wurde er dann, so erzählte er selber höchst zeitspezifisch im Ewigwährenden Calender, durch hessische Soldaten gefangengenommen und nach Kassel verschleppt. ${ }^{5}$ Im Mai 1636 nahm er, jetzt auf kaiserlicher Seite, an der Belagerung Magdeburgs und im Oktober desselben Jahres an der Schlacht bei Wittstock teil. Die verheerende Niederlage der kaiserlichen Armee dürfte zur Versprengung des Jungen nach Westfalen geführt haben, wo er 1637

${ }^{3}$ G. Könnecke, Quellen und Forschungen zur Lebensgeschichte Grimmelshausens (Weimar: Hrsg. J. H. Scholte, 1926)

${ }^{4}$ G. Könnecke, "Der Dichter und sein Held: Hanau - Kroatenlager - Kassel," in G. Weydt, Der Simplicissimusdichter und sein Werk (Darmstadt: Wissenschaftliche Buchgesellschaft, 1969), 34

Siehe auch Simplicissimus II, 14

5J. Petersen, "Hans Jacob Christoph von Grimmelshausen," in G. Weydt, Der Simplicissimusdichter, 57 
im Dragonerregiment des kaiserlichen Feldmarschalls Graf Hans von Götz wieder auftaucht.

Mit dieser Einheit kam der nunmehr 16jährige 1638 an den Oberrhein, um die von Bernhard von Sachsen-Weimar belagerte Festung Breisach zu entsetzen. Die nur mühsam zu verfolgende Spur des jungen Landsknechten begann sich im Jahre 1639 zu verdeutlichen, da sie ab diesem Jahr dokumentarisch $\mathrm{zu}$ beweisen ist. Er fand Unterkunft und Sold in der Reichsstadt Offenburg im neugeworbenen Regiment des Freiherrn Hans Reinhard von Schauenburg, zuerst als Musketier, doch 1640 schon als Regimentsschreiber. Seine eher lückenhafte Ausbildung dürfte auf der Schauenburgischen Regimentskanzlei durch den ihm vorgesetzten Magister Johannes Witsch vervollständigt worden sein. In den nächsten 8 Jahren lernte Grimmelshausen nicht nur Soldlisten und Tabellen zu führen, sondern auch seine Erzähllust zu bändigen, in Worte zu kleiden und niederzuschreiben.

Nach einem kurzen Aufenthalt in der Regimentskanzlei des Obristen Johann Burkhard von Elten, einem Schwager des Offenburger Kommandanten, kehrte er 1649 in die Stadt am Rande des Schwarzwaldes zurück, wo er am 30. August Catharina Henninger, die Tochter eines Regimentskameraden, heiratete. Dies war der Abschluß seiner Militärlaufbahn und der Einstieg in ein bürgerlich seßhafteres Dasein. ${ }^{6}$ Er verblieb allerdings in den Diensten seines

\footnotetext{
${ }^{6}$ Günther Weydt, Hans Jacob Christoph von Grimmelshausen (Stuttgart: Metzlersche Verlagsbuchhandlung, 1971), 8
} 
bisherigen Brotherrn, Hans Reinhard von Schauenburg, und dessen Vetter Carl Bernhard, deren weitverzweigten Familienbesitz er als Schaffner, einer Art Finanzbeamter, zu verwalten half. Die nächsten 10 Jahre, bis 1661, lebte er in Gaisbach, einem Dorf am Westrand des Schwarzwaldes, von wo er auf Dienstreisen die benachbarten Gebiete, das Tal der ungezähmt dahinfließenden Rench und die Renchtalbäder Petersthal und Bad Griesbach sowie die wichtige Kulturstadt Straßburg kennenlernte. Diese genauen Ortskenntnisse kamen ihm bei seinen Werken, deren Geschehen er hauptsächlich in diesem Gebiet ansiedelte, zugute.

Die Dienste der Schauenburger verließ er 1661 und fand kurze Zeit später Anstellung als Burgvogt in der dem damals berühmten Arzt Dr. Johannes Küffer gehörenden Ullenburg bei Gaisbach. Das Verhältnis zu dem Modearzt dürfte aber nicht sehr harmonisch gewesen sein, denn Grimmelshausen blieb nur drei Jahre in dieser Stellung. ${ }^{7} 1665$ eröffnete er in seinem kleinen Haus in Gaisbach einen Weinausschank, "Zum Silbernen Stern". Gleichzeitig wird er sich auch mit Macht auf seine literarischen Arbeiten gestürzt haben, denn schon zwei Jahre später erschienen seine ersten beiden Werke, Der satyrische Pilgram und Der keusche Joseph, die er unter dem Pseudonym Samuel Greifson von Hirschfeld herausbrachte. Im gleichen Jahr, 1667, bewarb er sich um die Stelle als Bürgermeister des kleinen Schwarzwaldortes Renchen, der nur wenige Kilometer

${ }^{7} \mathrm{G}$. Weydt, Hans Jacob Christoph von Grimmelshausen, 11 
entfernt am Ausgang des Renchbachtales gelegen ist. Mit finanzieller Unterstützung seines Schwiegervaters, der es mittlerweile zum Ratsherrn gebracht hatte, bekam Grimmelshausen die dem Bischof von Straßburg unterstehende Amtsstelle.

Nun fand sich der ehemalige Musketier in einer Rang und Würde bedeutenden Obrigkeitsfunktion, in der er sich fortan um öffentliche Ordnung und Verwaltung zu kümmern hatte. Aufgefundene Akten zeigen ihn als Gerichtsbarkeit bei Strafverhandlungen wegen unerlaubten Weinzapfens, bei Eheverträgen und als Abgabeneintreiber. ${ }^{8}$ Neben diesen gewichtigen und sicherlich auch zeitraubenden Aktivitäten produzierte er nun auch die Flut seiner literarischen Werke, beginnend $1668 \mathrm{mit}$ dem $\underline{\text { Simplicissimus }}$ bis zu seiner letzten Veröffentlichung im Jahre 1675, dem Wunderbarlichen Vogelnest II.

Aber noch einmal wurde der alternde Landsknecht vom Kriegsgeschehen des turbulenten 17. Jahrhunderts eingeholt. Der französische Marschall Turenne setzte 1673 im Rahmen des holländischfranzösischen Krieges über den Rhein, besetzte die Orte des westlichen Schwarzwaldes, auch Renchen gehörte dazu, und machte sie tributpflichtig.

Der Renchener Schultheiß befand sich sicher in einem Gewissenskonflikt, seinen ohnehin armen Bauern die Kontributionen für die Franzosen abzuverlangen, aber als Vertreter des Gesetzes blieb ihm keine andere Wahl. Einige Quellen vermuten, daß er sich an die

${ }^{8}$ V. Meid, 80 
Spitze einer Bürgerwehr setzte, um das Land der Bauern gegen die französischen Eindringlinge zu verteidigen. 9 Trotz der kriegerischen letzten Jahre seines Lebens verstarb der SimplicissimusDichter aber scheinbar friedlich am 17. August 1676 im Alter von 55 Jahren. Verschiedentlich hatte er seit 1671 Andeutungen auf eine Krankheit gemacht, aber es liegen keine Unterlagen darüber vor, daß er einer Krankheit zum Opfer fiel. Im Kirchenbuch Renchens findet sich lediglich die routinemäßige Notiz, daß es sich bei dem Verstorbenen um einen gebildeten und hoch angesehenen Mann gehandelt hatte.10 1876, zu seinem zweihundertsten Todestag, errichtete die Gemeinde allerdings ein Denkmal zu Ehren ihres berühmtesten Bürgermeisters.

${ }^{9}$ Herbert Heckmann,"Grimmelshausen, oder die Leiden eines Volksschriftstellers," in W. Hinderer, Literarische Profile - Deutsche Dichter von Grimmelshausen bis Brecht (Königstein: Athenäum Verlag, 1982), 19

${ }^{10} \mathrm{Ch}$. Stoll, Hans Jacob Christoffel von Grimmelshausen (München: Heinz Moos Verlag, 1976), 5 


\section{KAPITEL}

\section{DIE ENTSTEHUNG DES WERKES}

Bei genauer Analyse der Werke Grimmelshausens lassen sich drei Themenkreise herauskristallisieren. Neben dem vom Dichter selbst ernannten Simplicissimus-Zyklus, dem außer dem Hauptwerk noch die Landstörzerin Courasche, der Springinsfeld Roman, sowie die beiden Teile des Wunderbarlichen Vogelnest angehören, versuchte sich der schreibende Bürgermeister noch in der Abfassung von Kalendergeschichten, die sich $\mathrm{zu}$ der damaligen Zeit enormer Popularität erfreuten. Darin wurden in anekdotischer Form Sprüche, Kurzgeschichten und Situationen dargestellt, die die Leserschaft unterhalten und belehren sollten. Grimmelshausen folgte dabei allerdings nicht dem herkömmlichen Stil des unpersönlichen Erzählens, sondern er versuchte, die Geschichten durch seine bekannten simplicianischen Figuren zu koordinieren und ihnen einen zeitlichen und persönlichen Rahmen zu verleihen. ${ }^{1}$

Den dritten Zweig seines schriftstellerischen Schaffens stellen die beiden höfisch-heroischen Romane Dietwald und Amelinde und Proximus und Lympida dar, die aber verkaufsmässig als Mißerfolg zu gelten haben. In diesen Werken versuchte sich der Dichter in

1V. Meid, 174 
der Gattung des klassischen Barockromans, der zu seiner Zeit so überaus beliebt war, aber dessen schwulstiger Stil seiner volkstümlichen Hand wohl wenig behagte.

Zwar gibt die genaue Festlegung der Veröffentlichungsdaten des Simplicissimus der Philologie auch heute noch Rätsel auf, in der Reihenfolge der Werke Grimmelshausens hingegen herrscht weitgehende Übereinstimmung. Zweifellos begann er während seiner Militärzeit als Regimentsschreiber in Offenburg schon mit der Aufzeichnung von Beobachtungen und erinnerungswürdigen Notizen. So findet sich am Schluß der "Continuatio" die Bemerkung, daß er den Simplicissimus zu einer Zeit geschrieben habe, als er noch "ein Musquetier" gewesen war.

Das Manuskript des Romans dürfte schon früh im Jahre 1668 in den Händen des Nürnberger Verlegers Wolff Eberhard Felßecker gewesen sein, denn eine Anzeige im Ostermeßkatalog von 1668 weist auf die baldige Veröffentlichung des Buches hin. ${ }^{2}$ Grimmelshausen machte im 1673 erschienen Teutschen Michel darauf aufmerksam, daß er sein Hauptwerk "auf einen Wurf" geboren habe. ${ }^{3}$ Es erscheint als wahrscheinlich, daß er damit die letzte Überarbeitung des Stoffes im Jahre 1667 vor der Übergabe an seinen Verleger gemeint hat, denn es wird als gegeben angenommen, $\mathrm{da} B$ er den Ewigwährenden Calender, der erst 1671 erschien, schon

2 Manfred Koschlig, "Grimmelshausens Schriften in den Meßkatalogen 1665-1675," in G. Weydt, Der Simplicissimusdichter. 105

${ }^{3}$ Grimmelshausen, Simplicianische Schriften (München: Winkler Verlag, 1958), 720 
vor dem Simplicissimus, zumindest aber gleichzeitig, bearbeitet hat. ${ }^{4}$ Die Forschung vermutet in dem Sammelband von literarischen Aufzeichnungen und selbstbiographischen Notizen eine Art Arbeitsbuch zur Erstellung des großen Romans.

Hier drängt sich die Frage nach der Arbeitsweise des Schwarzwälder Romanautoren förmlich in den Vordergrund. Wann hatte er die Zeit, neben seinem vollen Tagespensum als Schultheiß eines Dorfes, dem mehrere entlegen liegende Bauerngüter und Weiler angehörten, und den Pflichten seiner vielköpfigen Familie gegenüber, er war der Vater von immerhin zehn Kindern, sich auf das Schreiben von, wie heute vermutet wird, mindestens zwanzig literarischen Werken zu konzentrieren? Die Antwort auf diese Frage läßt sich, wie so vieles aus dem Leben und Schaffen Hans Jacob Christoph von Grimmelshausens, nur noch erahnen.

In schneller Reihenfolge flossen nach dem Simplicissimus eine Reihe anderer simplicianischer Schriften aus des Dichters Hand. Im Jahre 1670 erschien Trutz Simplex: Die Lebensbeschreibung der Landstörzerin Courasche, gefolgt von Der Seltzame Springinsfeld 1671. Die Reihe der simplicianischen Schriften setzte sich 1672 mit dem Wunderbarlichen Vogelnest fort, dem nach einer dreijährigen Pause 1675 Das Wunderbarliche Vogelnest Teil II folgte, das Grimmelshausens letzte Veröffentlichung war. Dazwischen schob der emsige Schultheiß eine Anzahl von Kalendergeschichten und nebensächlichen Aufsätzen, sowie die beiden bereits genann-

${ }^{4}$ G. Weydt, Hans Jacob Christoph von Grimmelshausen, 36 
ten heroischen Romane.

Die Handlung des Simplicissimus, die hier als hinlänglich bekannt vorausgesetzt wird, läuft in vieler Hinsicht parallel zu dem Leben des Autoren, unterscheidet sich aber doch in wesentlichen Teilen davon. So belegte Könnecke eindeutig, daß der Rahmen und der allgemeine Verlauf des Romans von Grimmelshausen selbstbiographisch gestaltet wurde, viele Einzelheiten jedoch historischen Quellen und Nachschlagewerken entnommen wurden. Es ist dabei aufschlußreich zu sehen, wie der Dichter bei der Vereinigung von Realität und Phantasie zu Werke ging. Darüber wird im Kapitel 5 noch mehr zu sagen sein.

Sicher ist, daß Grimmelshausen sich bewußt oder unbewußt von jeglichen Vorbildern trennte und an keine bestimmte Literaturform binden wollte. 5 Die unkonventionelle Behandlung des Stoffes, ebenso wie die Auswahl der Materie, hält den Leser in Atem und gibt der Forschung zu immer neuen Vermutungen Anlaß. Die Rätsel, die dieser ungewöhnliche Barockroman schon seiner Mit-, ganz besonders aber seiner Nachwelt aufgab, sind wahrhaftig einzigartig in der deutschen Literatur. Schon der Name des Verfassers, "German Schleifheim von Sulsfort", verwirrte die Fachwelt bis in die Mitte des 19. Jahrhunderts. Auch der Verlagsort "Mönpelgart" und der angegebene Name des Verlegers, "Johann Fillion", geben immer noch Stoff für literarische Debatten ab. So glaubt Scholte in der Auswahl der protestantischen Hochburg Mönpelgart, einem Städtchen im 
Elsaß, das dem Herzog von Württemberg gehörte und Ort der 1586 stattgefundenen Religionsdiskussion zwischen Kalvinisten und Lutheranern gewesen war, einen Hinweis dafür sehen zu können, mit dem Simplicissimus eine Religionssatire vor sich zu haben. ${ }^{6}$ Diese Annahme wird von der neueren Forschung aber als unzulänglich nachweisbar abgelehnt. ${ }^{7}$ Es war im 17. Jahrhundert, einer Zeit der strengen Zensur von Seiten der Kirche und der Territorialstaaten, keine Seltenheit, den Ersteller einer Schrift hinter einem fingierten Namen zu verbergen. Warum aber der Verleger sowie der Verlagsort des Simplicissimus so gründlich vertuscht worden sind, ist auch heute noch unbekannt.

Gedruckt wurde die Erstausgabe des Buches 1668, aber schon ein Jahr später schob der Verleger Felßecker eine neue Auflage hinterher, die zu Ostern 1669 erschien und die um die "Continuatio" erweitert worden war. Im Herbst 1668 war die deutsche Übersetzung der englischen Robinsonade The Isle of Pines von Henri Neville erschienen, dessen Inhalt Grimmelshausen wohl Anregungsmaterial für seine "Continuatio" geboten hatte.

Im Verlauf der nächsten Jahre erlebte der Simplicissimus eine Popularität, wie sie nur wenigen Werken der deutschen Literatur vergönnt ist. Ähnlich wie Schillers Räuber und Goethes Werther fanden die verschiedenen Ausgaben des Simplicissimus ein derart

6 J. H. Scholte, 40

${ }^{7}$ G. Weydt, Nachahmung und Schöpfung im Barock (Bern: Francke Verlag 1968), 198 
begeistertes Publikum, daß der Verleger sich genötigt sah, noch weitere Auflagen in dichter Folge zu drucken. Allerdings war auch die Konkurrenz aufmerksam geworden. Schon im Herbst 1669 erschien durch den Frankfurter Verleger Georg Müller ein Raubdruck, der sich zwar sprachlich erheblich von dem Original abhob, aber dennoch Einfluß auf spätere Editionen genommen hat. ${ }^{8}$ Der Frankfurter Simplicissimus unterschied sich von dem Original hauptsächlich durch eine Glättung der mundartlichen Sprache und durch Tilgung von grammatischen Unebenheiten, und wird daher von der Forschung als "Schulmeister" Simplicissimus klassifiziert. Für die Forschungsgeschichte des Buches ist diese Ausgabe nur von sekundärer Bedeutung. 9

Die von der außerordentlichen Nachfrage geschürten Neuauflagen des Romans schaffen eine heillose Verwirrung in der Festlegung der von Grimmelshausen persönlich überarbeiteten Ausgaben seines Werkes. Auch er versuchte vermutlich sein Buch nach dem erstaunlichen Anfangserfolg so verkaufsfreundlich wie möglich zu gestalten. Aus diesem Grunde erschienen die nächsten beiden Neuauflagen im Jahre 1671 mit einer Vielzahl von Abbildungen, die vermutlich aus des Dichters Hand selber stammten. Auch die sprachlichen Veränderungen, die sich an der Frankfurter Ausgabe orientierten, sollten wohl dazu dienen, das Buch weiteren Bevölkerungsschichten zugängig zu machen.

${ }^{8} \mathrm{G}$. Weydt, Hans Jacob Christoffel von Grimmelshausen, 18

9J. H. Scholte, 209 
$\mathrm{Da} \beta$ die Geschichte des Simplicius die begüterte Klasse des 17. Jahrhunderts unterhalten würde, war wohl zu erwarten gewesen. Die Herzogin Sophie von Hannover schrieb schon 1670 an ihren Bruder Karl Ludwig von der Pfalz von ihrem Gefallen an den Abenteuern des Simplicissimus. Auch der Mathematiker und Philosoph Gottfried Wilhelm von Leibniz berichtete von der vergnüglichen Lektüre des Romans, den er dem französischen "Francion" gleichstellte. 10 Gemeint war damit wohl der Schelmenroman Histoire Comique de Francion des Charles Sorel, der 1623 geschrieben und 1662 ins Deutsche übersetzt worden war.

Es ist leicht zu verstehen, daß sich der Markt für die zeitgenössische Literatur des 17. Jahrhunderts bei den Aristokraten und Universitätsabsolventen fand. Für die lesebedürftigen Kleinbürger jener Zeit, die durchaus als potentielle Käufer von Büchern anzusehen sind, stand der enorm hohe Kaufpreis als fast unüberwindliches Hindernis im Weg. So kostete der 1800 Seiten starke heroische Roman Arminius des Daniel Caspar von Lohenstein 8 Reichstaler, was etwa dem Preis von $130 \mathrm{~kg}$ Rindfleisch entsprach. ${ }^{11}$ Bücher waren für den gemeinen Mann unerschwinglich teuer. Obwohl der Simplicissimus nicht den Umfang des Lohensteinschen Epos' hatte, dürfte sein Preis etwa um einen halben Reichstaler gelegen haben, wofür ein Kaufmann, Apotheker oder

${ }^{10} \mathrm{Ch}$. Stoll, Hans Jacob Christoffel von Grimmelshausen, 71

${ }^{11}$ A. Martino, "Barockpoesie, Publikum und Verbürgerlichung der literarischen Intelligenz," Internationales Archiv für Sozialgeschichte der deutschen Literatur, 1, (1976), 107 
Schreiber doch immerhin eine halbe Woche arbeiten mußte. 12

Umso erstaunlicher ist der Verkaufserfolg des Grimmelshausenwerkes, wenngleich Volker Meid die Gesamtauflage zur Lebzeit des Autoren auf nur etwa 10.000 bis 12.000 Exemplare schätzt, was nach heutigen Verhältnissen als lächerlich gering anmutet. ${ }^{13}$ Der Erfolg des Werkes eines unbedeutenden und darüberhinaus unbekannten Schwarzwaldautoren war jedoch ein Zeichen für die literarische und gesellschaftliche Umwälzung, die sich im deutschsprachigen Raum zur Mitte des 17. Jahrhunderts anzubahnen begann.

12H. D. Gebauer, 412

13V. Meid, 64 


\section{KAPITEL \\ DIE INTERPRETATION DES ROMANS \\ DURCH DIE FORSCHUNG}

Nachdem 1713 noch einmal eine letzte Gesamtausgabe der simplicianischen Schriften gedruckt worden war, geriet der Simplicissimus und sein Dichter fast für ein Jahrhundert in Vergessenheit. Zwar erwähnte ihn Lessing in einer Notiz, die 1787 post hum im Allgemeinen Gelehrten-Lexikon erschien. Darin bezeugte er aber hauptsächlich nur, wie wenig über den Autor des Barockromans noch in Erinnerung geblieben war:

Greifensohn, (Samuel,) aus Hirschfeld, lebte im vorigen Jahrhundert, und war in seiner Jugend Musketier. Mehr ist nicht von ihm bekannt, ob er gleich verschiedenes geschrieben hat; nehmlich: Den Simplicissimus, einen zu seiner Zeit beliebten Roman, welchen er anfänglich unter dem versetzten Namen German Schleifheim von Sulsfort herausgab $\ldots 1$

Den Dichtern der Romantik, und da besonders Ludwig Tieck,

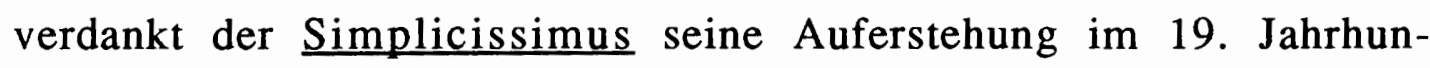
dert. Tieck übernahm in seiner Erzählung Das Tagebuch im Jahre 1798 einen Teil der Jupiter-Episode, deren politische Aussage zur Zeit der Napoleonischen Kriege eine unerwartete Aktualität be-

${ }^{1}$ G. Weydt, Nachahmung, 12 
kam. Angeregt durch Tiecks Schrift erschien 1809 eine ausführlich kommentierte Fassung der Jupiter-Episode von einem anonymen Schriftsteller, in der dieser den Zerfall und die Ohnmacht des Deutschen Reiches beklagte. Achim von Arnim und Clemens Brentano nahmen das Nachtigallen-Lied in ihrem Wunderhorn auf, und Tiecks Freund Karl Eduard von Bülow gab 1836 eine neue Bearbeitung des Hauptwerkes heraus: Die Abenteuer des Simplicissimus. Ein Roman aus der Zeit des Dreißigjährigen Krieges.

Bülow bezeichnete das Werk zwar als "den ersten Original Roman" der deutschen Literatur, aber er sah in ihm doch nur ein "Volksbuch" von historischem Wert. ${ }^{2}$ Dieses Interpretationsbild sollte noch für Jahrzehnte die Forschung beeinflußen. Man ging von der Voraussetzung aus, in Grimmelshausen, dessen wahrer Name 1837 entlarvt wurde, einen Autor mit gesundem Menschenverstand und derbem Humor zu sehen, der aber als urwüchsiges Erzählertalent doch nicht auf der Höhe der Bildung seiner Zeit gestanden hatte. ${ }^{3}$ Die sich im Laufe des 19. Jahrhunderts als Wissenschaft entwickelnde Germanistik stufte das Werk als "Volksbuch" und dessen Schriftsteller als "Volksdichter" par excellence ein, der sich vor allem in der realistischen Beschreibung des Lebens seiner Zeit ausgezeichnet hatte. 4

2V. Meid, 217

${ }^{3}$ G.Weydt, Nachahmung, 13

${ }^{4}$ V. Meid, 219 
Die Entstehung des Deutschen Reiches unter preußischer Herrschaft brachte dem Simplicissimus 1876 allerdings scharfe Kritik von Seiten der deutsch-nationalistischen Parteien im preußischen Abgeordnetenhaus ein. Der Zentrumsabgeordnete Freiherr von Schorlemer-Alst warf dem Buch Grimmelshausens einen politisch und moralisch untragbaren Standpunkt vor:

Ich habe in dem ganzen Buche darauf nachgesehen, es durchgelesen, zu finden wo denn etwas zur Weckung der vaterländischen Gesinnung stände. Das Gegentheil habe ich wohl darin gefunden; denn als der Simplicius Simplicissimus sich endlich entschließt auf vieles Zureden seines alten Kriegskameraden, ein Raubmörder und Wegelagerer zu werden, da überredet ihn der alte Kumpan mit den Worten: das Raubhandwerk wäre das alleradeligste Handwerk, denn das trieben selbst die Könige, indem sie ihren Unterthanen die Steuern auspressen. ${ }^{5}$

Die Grundeinstellung des Dichters ließe eine Verteilung seines Werkes im neugeformten Deutschen Reich einfach nicht zu:

Jawohl, meine Herren, wenn man den Kindern Bücher in die Hände gibt, die einen derartigen Inhalt haben, so regt man sie zu weiteren Nachdenken nach der Richtung und zum weiteren Forschen an, und das ist seelenmörderisch, und das spreche ich vor dem ganzen Lande aus. ${ }^{6}$

Die "Grimmelshausen-Debatte", die sich über zwei Tage hinzog, konzentrierte sich neben der sozialkritischen Perspektive auf die religiöse Bedeutung des Buches. So befand der Führer des Zentrums, Ludwig Windthorst, daß "wer einen bitteren $\mathrm{Ha}$ gegen die

5H. D. Gebauer, I

$6_{\text {ebda, III }}$ 
Katholiken verbreiten will, der muß dieses Buch lesen, der muß es verbreiten,... ${ }^{7}$

Im frühen 20. Jahrhundert versuchte die Grimmelshausen-Forschung hauptsächlich, Licht in das geheimnisvolle Leben des Autors zu bringen. Das ausschlaggebende Werk Gustav Könneckes in dieser Richtung wurde schon im 2. Kapitel vermerkt. Mit der Einsicht, daß das Leben des Autors sich nur bedingt im Simplicissimus widerspiegelt, eröffneten sich neue Interpretationsschemen. Der Roman verlor allmählich seinen Ruf als das Erzeugnis eines schlichten Volksdichters, und erhielt fortan eine Vielzahl von Auslegungsmöglichkeiten, die sich aber oftmals gegenseitig ausschloßen.

Johannes Alt erkannte als erster im Simplicissimus eine streng eingehaltene Struktur, die der Dichter durch eine Verquickung seines eigenen Lebens mit eingeschobenen Abenteuern und Legenden erreichte. Diese Struktur enthält fünf klar abgeteilte Stufen, die von "Gönner" über "Lehrer" zu "Selbstentfaltung" und "Gegenkräfte" kommen, bevor sich die Handlung jeweils in der Mitte des folgenden Buches "beruhigt". 8 Dieses Schema, so Alt, weist den Roman eindeutig als barocken Bildungsroman aus. Ähnlich wie Goethe Wilhelm Meister als einen die Gesellschaft transzendierenden Schauspieler schuf, so ließ Grimmelshausen seine Hauptperson als Soldat die Höhen und Tiefen seiner Umgebung erleben. Zwar kann man den $\underline{\text { Simplicissimus nicht direkt mit Wilhelm Meister }}$

7V. Meid, 228

8J. Alt, 201 
vergleichen, da die Motivierung zur Zeit Grimmelshausens in der Erlangung des Seelenheils ruhte, Goethe hingegen die Vollendung der Bildung und des Geistes anstrebte. Aber in beiden Geschichten gibt die Auseinandersetzung mit der Welt die Anstöße zur Fortentwicklung, wenngleich die Welt des 17. Jahrhunderts auch roh, verwirrt und zerrissen war, während die Zeit Goethes sich kulturell sittsam, geistig und gebildet gab.

Ganz entschieden gegen die Auffassung des $\underline{\text { Simplicissimus als }}$ Bildungsroman spricht sich hingegen Ernst Eugen Stark aus. ${ }^{9} \mathrm{Er}$ sieht die angeführten Entwicklungsstufen als Kennzeichen des Picaro-Romans im Allgemeinen an. Da jeder Schelmenroman dasselbe Schema durchliefe, wäre auch jeder Picaro-Roman ein Bildungsroman, was natürlich nicht der Fall sei. Auch sieht Stark keinerlei Entwicklung im Sinne einer langsamen seelischen Veränderung des Simplicius. Alle neuen Lebenserkenntnisse blieben episodenhafte Fragmente, die der Dichter bald schon wieder wechsele. Der Simplicissimus, dessen Held ziel-, sinn- und endlos vorwärts genau wie rückwärts treibe, stelle keine Entwicklung eines Menschen dar.

Auch Richard Alewyn hegt die Befürchtung, daß Grimmelshausen "nichts wollte, als erzählen und immer wieder erzählen" und dabei, fast als Nebenerscheinung, eine Gestalt geformt hat, die am Ende mehr Schichten in sich trug, als ihr jemals zugedacht

\footnotetext{
${ }^{9}$ Ernst Eugen Stark, "Barockprosa im Deutschunterricht," in Jentzsch, Brauneck, Stark, Das 17. Jahrhundert in neuer Sicht (Stuttgart: Ernst Klett Verlag, 1969), 47
} 
waren. 10

$\mathrm{Da}$ diese Debatte längst noch nicht abgeschlossen ist, beweist Peter Daly. In seinem 1985 erschienen Artikel behauptet er, in dem Simplicissimus, wenn schon keinen herkömmlichen Bildungsroman, so doch einen christlichen Bewährungsroman sehen zu können, der sich durch die Festlegung der christlichen Lebensmaßstäbe in den ersten zwölf Kapiteln des Buches ergibt. ${ }^{11}$ Das Vermächtnis des alten Einsiedels, Selbsterkenntnis, Beständigkeit und den richtigen Umgang im Leben, stelle das Bildungsprinzip dar, nach dem Simplicius sein Leben zu steuern versuche.

Auch andere Forscher machen auf den moralischen Wert der Werke Grimmelshausens aufmerksam. Joldersma und Wickert weisen auf die geglückte Verwirklichung von Unterhaltung und moralischer Belehrung hin, die durch die gewählte Ich-Form des Erzählers gelungen ist. ${ }^{12}$ Diese Ich-Perspektive ist auch einer der Gründe, den Simplicissimus in die Kategorie der PicaroRomane einzustufen.

Zwar war der Einfluß der spanischen Picaro-Romane auf Grimmelshausen schon im 19. Jahrhundert entdeckt worden, aber die

10 Richard Alewyn, "Grimmelshausen Probleme," in G. Weydt, Der Simplicissimusdichter, 398

${ }^{11}$ Peter Daly, "Simplicissimus Teutsch - doch ein Bildungsroman?" in Alte und neue Kontroversen, Akten des VII. Internationalen Germanistenkongresses Göttingen 1985 (Tübingen: Max Niemeyer Verlag, 1986), 159

12 Hermina Joldersma, Elke Wickert, "Moraldidaktik, Erzählform und Autorintention in Grimmelshausens Landstörzerin Courasche," The Germanic Review, v. 64, 4, (fall 1989), 158 
nationalistisch gesinnte Einstellung der Germanistik jener Zeit verbot es, diesen Einfluß offen anzuerkennen. In den Picaro-Romanen, in denen das Leben einer nicht eben heldenhaften Gestalt beschrieben wird, reihen sich die in der Ich-Form erzählten Abenteuer in chronologischer Reihenfolge aneinander. In diesen Schilderungen, die sich im barocken Deutschland großer Beliebtheit erfreuten, zeigen sich die krassen Gegensätze der Zeit besonders deutlich. Frommer Glaube steht offener Skepsis gegenüber, und strikte Askese muß sich gegen überschwengliches Genußtrachten behaupten.

Im Simplicissimus finden sich offensichtliche Charakteristiken der Schelmenromane. Einzelepisoden, wie die Speckdiebstahlgeschichte oder die Jupiter-Episode, folgen einander und werden durch eine derbe Wortwahl volksverbunden dargeboten. Im Gegensatz zum höfisch-heroischen Romantypus erzählt die Handlung von Personen niedriger Abstammung, wie Bauern, Soldaten oder Vagabunden, mit denen sich der Leser durch deren realistisches Verhalten identifizieren kann. So ist es nicht verwunderlich, daß Grimmelshausens Hauptwerk mancherorts als "der Gipfel des pikaresken Romans in Deutschland" gepriesen wird. 13

Grimmelshausen beschränkte sich jedoch nicht auf eine einfache episodenhafte Aneinanderreihung von Abenteuern. Vielmehr wob er die einzelnen Elemente, wie Herzbruder, Olivier oder den Knan,

${ }^{13}$ Herbert Singer, Der galante Roman (Stuttgart: Metzlersche Verlagsbuchahndlung, 1961), 15 
kunstvoll zusammen, wobei er verschiedene Literaturstile überbrückte. Mit der adeligen Abstammung seines Helden verlieh er seinem Werk sogar den Hauch einer höfisch-heroischen Form, die diametral dem Schelmenroman gegenüber steht. Diese Formenverschmelzungen mögen ein Beweis für Grimmelshausens literarische Ignoranz sein, wie einige Kritiker, unter ihnen Clemens Lugowski, beharrlich dem Schwarzwaldautor unterstellen. ${ }^{14}$ Sicher ist, da $B$ der Dichter sich an keine etablierten Modelle hielt, aber dafür ein umso reichhaltigeres Werk geschaffen hat.

Wieviel die Forschung in dem Simplicissimus zu lesen im Stande ist, beweisen die Aufschlüsselungen der astrologischen Strukturen des Romans durch Günter Weydt.15 Der eminente GrimmelshausenKenner glaubt durch ein Deuten der astrologischen Zeichen, die sich hauptsächlich im Ewigwährenden Calender finden, das Dunkel um die Autorenintention beim Simplicissimus erhellen zu können. Daß Grimmelshausen mit der Planetensymbolik vertraut war, zeigte er im Ewigwährenden Calender sowie in der Jupiter-Episode hinlänglich genug. Weydt benutzt einen im Calender gegebenen Hinweis, daß Jupiter geographisch über Köln herrscht, als Dreh- und Angelpunkt seiner tiefsinnigen Theorie.

Simplicius geht am Ende des dritten Buches nach der JupiterEpisode zu einem Ort, den der Dichter in der Kapitelüberschrift

${ }^{14}$ Clemens Lugowski, "Literarische Formen und lebendiger Gehalt im Simplicissimus," in G. Weydt, Der Simplicissimusdichter, 170

15 siehe G. Weydt, Nachahmung, $243-279$ 
"pro forma Cöln" nannte, wobei sich bei der Wegbeschreibung allerdings keine Zweifel über die tatsächliche Ankunft des wackeren "Jäger von Soest" in der Rheinmetropole erlauben lassen. Die geheimnisvolle Redewendung in der Kapitelüberschrift glaubt Weydt als Zeichen Grimmelshausen bewerten zu können, die unterschwellige Verknüpfung des Simplicius' mit dem Planetengott Jupiter, der ihm Macht und Ansehen gebracht hat, hier zu beenden. Wahrhaftig wendet sich das Glück des bis dahin über grosse Reichtümer verfügenden Simplicius' von nun an. Ähnliche Verbindungen erkennt Weydt im Leben des Buchhelden mit den anderen Planeten Saturn, Venus, Mars, Merkur, Sonne und Mond. Diese faszinierende Symbolik wirft ein neues Licht auf die kunstvolle Gestaltung des Barockromans, die weit über das lang geglaubte "erzählen und immer noch mehr erzählen" des Simplicissimus-Dichters hinausgeht.

Die Vielzahl der Interpretationsmodelle gliedern sich heute hauptsächlich in drei Sachgebiete auf:

1. Die symbolisch-allegorische Richtung.

Hierzu gehören die Planetensymbolik und die christlich-typologische Deutung.

2. Die emblematische Richtung. Hier vor allem die Titelkupfer.

3. Die sozial- und kulturgeschichtliche Richtung.

Die Bauerndarstellung sowie die kulturgeschichtlichen Hintergründe 
und die Verwertung ausländischer Stoffe stehen hier im Mittelpunkt. ${ }^{16}$

Obwohl ein endgültiges Verständnis des Simplicissimus unter der Flut der Auslegungen in absehbarer Zeit noch nicht sichtbar ist, beweisen eben diese unterschiedlichen Interpretationsmöglichkeiten, welch ein vielschichtiges Meisterwerk der Renchener Schultheiß vor mehr als 300 Jahren geschaffen hat. 


\section{DIE PERSÖNLICHKEIT DES DICHTERS}

Die menschliche Seite eines berühmten Schriftstellers ist für die meisten Leser höchstens aus Neugierde, und für die Literaturforschung lediglich zur Abrundung des Gesamtbildes des Autoren wichtig. In vielen Fällen lassen sich jedoch Beweggründe und Motivation eines Dichters für ein bestimmtes Werk aus seiner Persönlichkeit und seiner Einstellung ermitteln. Die kreativen Leistungen eines Menschen stehen oftmals in engster Verbindung mit seinen Lebensanschauungen, wenn es auch höchst fahrlässig wäre, die veröffentlichten Schriften immer als die persönliche Meinung des Autoren zu bewerten. Von den meisten deutschen Schriftstellern und Dichtern ist solch ein Persönlichkeitsprofil kein sehr schwieriges Unterfangen, da normalerweise zahlreiche Briefe, Tagebuchaufzeichnungen und aufgeschriebene Diskussionen oder Unterhaltungen der betreffenden Person erhalten sind. Das ist allerdings im Fall Grimmelshausen nicht, oder nur sehr bedingt, zutreffend.

Eine geheimnisvolle Person wie die des Simplicissimusautoren fordert die Spürsinne des Forschers geradezu heraus, näheres über ihn ausfindig zu machen. Wie schon an mehreren Stellen dieser Arbeit hervorgehoben wurde, fehlt es an ungelösten Rätseln um 
den Renchener Bürgermeister wahrhaftig nicht. Allerdings kann eine Nachforschung über sein Verhalten, seine Denk- und Arbeitsweise, seine Weltanschauung, kurz, über seine Persönlichkeit nur durch ein genaues Begutachten seiner Bücher erfolgen, da weder persönliche Briefe noch Beobachtungen seiner Zeitgenossen erhalten blieben. Die Gefahr einer subjektiven, fehlgeleiteten Persönlichkeitsbeschreibung liegt also auf der Hand. Andererseits ist sich die Forschung einig darüber, daß Grimmelshausens Werke sehr stark von der persönlichen Meinung des Dichters geprägt wurden, so daß sie also durchaus als Quellenmaterial in Frage kommen können. Zwar stellen die neueren Formen der Literaturkritik für Interpretationszwecke die Verbindung zwischen Autor und Werk in Frage, aber, wie Gary Stark in einem aufschlußreichen Artikel ausführte, bildet die Literatur einer Zeit eine Brücke zur Denkweise der Menschen jener Zeit, und somit auch zu den Gedankengängen des Autoren. I

Dabei ist es wesentlich festzustellen, daß eine solche Untersuchung nur über die Einstellungen Grimmelshausens einigermaßen sichere Auskunft geben kann, sofern sie sich in Stellungsnahmen und Kommentaren in seinen Büchern widerspiegeln. Über seine psychischen Merkmale oder seine gemütsbedingten Reaktionen läßt sich nur weniges, und das nur hypothetisch ableiten.

Als Endziel soll hier eine möglichst breite Aufstellung von wichtigen Einzelmerkmalen anvisiert werden, die sich dann zu einer

${ }^{1}$ Gary Stark, "Vom Nutzen und Nachteil der Literatur für die Geschichtswissenschaft," in German Quarterly (winter 1991), 27 
Einheit der kreativen Person Grimmelshausens zusammenfassen lassen. Das so erhaltene Bild der literarischen Persönlichkeit soll dann im folgenden Kapitel bei der Beantwortung der Frage nach dem Grund für die Entstehung des Simplicissimus Hilfestellung leisten. Ein ähnliches Unterfangen hat 1924 Rudolf Lochner bereits veröffentlicht, bei dem er sich allerdings auf eine reine Persönlichkeitsanalyse beschränkte, ohne auf die Beweggründe für das literarische Streben des Dichters einzugehen. ${ }^{2}$ Diese Arbeit wurde hier berücksichtigt.

Für Grimmelshausens vorzügliche Beobachtungsgabe finden sich mannigfaltige Belege in allen seinen Werken. Er war zwar in seiner Bodenständigkeit verwurzelt, behielt aber ein offenes Auge für das öffentliche und private Gesellschaftsleben um ihn her. ${ }^{3}$ In der Beschreibung der verschiedenen Schichten seiner zeitgenössischen Gesellschaft schillert die Erzählkunst Grimmelshausens am kräftigsten. Hervorgerufen durch die Schrecken des langen Krieges zeichnete sich die Barockgesellschaft des 17. Jahrhunderts durch eine immer weiter um sich greifende Verrohung im zwischenmenschlichen Umgang aus. Gleichzeitig begann aber eine Verschleierung der wahren Absichten durch übertriebene Höflichkeitsfloskeln, geheuchelte Ehrerbietung und falsche Galanterie, die die Wahrung des Scheins als oberste Priorität ansah. Der Adel ahmte

${ }^{2}$ Rudolf Lochner, Grimmelshausen, ein deutscher Mensch im 17. Jahrhundert Prager deutsche Studien (Reichenberg: Sudetendeutscher Verlag Franz Kraus, 1924)

${ }^{3}$ J. Petersen, 58 
immer mehr das Modell der französischen Aristokratie in Manieren, Sitten, Kleidung und Sprechweise nach, während die niederen Bürger alle Macht aufboten, in nichts nachzustehen.

Grimmelshausen zog in seinen Büchern immer wieder gegen die Falschheit und Heuchelei seiner Zeit zu Felde. Besonders spezifische Beispiele finden sich im Wunderbarlichen Vogelnest aber auch im Simplicissimus. Die berühmte Mummelsee - Szene zeichnet die Barockgesellschaft mit unverhohlener Ironie. Die Geistlichen stehen über allen Lastern, Kaufleute bedienen neid- und geizlos ihre Kunden, Wirte erquicken aus selbstloser Barmherzigkeit ihre durstigen Gäste mit Wein, Ärzte und Apotheker haben nur die Gesundheit ihrer Patienten im Auge, Handwerker kennen bei ihren Reparaturen weder Lüge noch Schein, den Armen wird von den Reichen zinslos genug zum Leben gegeben, denn jeder erkennt, daß einjeder als Ebenbild Gottes geschaffen wurde. ${ }^{4}$ Welch ein Hohn schwingt in dieser Beschreibung der Zustände in der menschlichen Gesellschaft mit!

Gegen bestimmte Berufssparten zog der Schultheiß aus dem Renchtal besonders vom Leder. So bezichtigte er die Rechtsanwälte rundweg des Wuchers und der Halsabschneiderei. 5 Sein berühmtes Portrait des selbstherrlichen Dr. Canard in Paris, der sich nur um reiche Adelige kümmerte, deren Krankheiten er zu seinen Gunsten ausnutzte, und der nebenbei gutaussehende Jünglinge an die ge-

${ }^{4}$ Simplicissimus V, 15

5 ebda III, 23 
langweilten Damen des Hofes verkuppelte, wird von der Forschung nicht nur als Spitze gegen den Ärztestand angesehen, sondern auch als Hinweis auf Grimmelshausens schlechtes Verhältnis zu seinem Arbeitgeber auf der Ullenburg, Dr. Küffer, gedeutet. ${ }^{6}$ Simplicius' eigener Versuch als reisender Quacksalber wurde von Grimmelshausen ebenfalls so ausführlich geschildert, daß dem Leser keine Zweifel über die zwielichtige Kunst der Ärzte bleiben können.7

Auch das Bild der Geistlichkeit wurde von der Feder des $\mathrm{Au}$ toren in nicht eben günstigen Farben gemalt. Der Hanauer Pfarrer lehnt es opportunistisch ab, gegen die wilde Zecherei beim Gouverneur der Festung zu predigen ${ }^{8}$, er trinkt später sogar mit und wirft den jungen Simplicius wenig später aus seinem Haus, weil der die Gunst des Gouverneurs zu verlieren droht. ${ }^{9}$

Bei alledem unterschied der Schreiber doch zwischen den niederen, schwachen und den edlen, barmherzigen Seiten des Menschen. Er verlor das Lächeln trotz der erhellten Mißstände nicht, denn seine verschmitzte Ironie trug ihn stets weit über diese beklagenswerten Zustände hinaus. Es ist leicht vorstellbar, daß der Schwarzwalddichter zwar traurig über die schlimmen Zustände seiner Zeit, aber nicht vollends vergrämt oder gar ver-

${ }^{6}$ Simplicissimus IV, 3

7 ebda IV, 8

8 ebda I, 30

$9_{\text {ebda II, } 2}$ 
bittert war. ${ }^{10}$

Die ausschweifenden Sitten und die sinkenden moralischen

Werte seiner Mitmenschen prangerte Grimmelshausen unabläßig an. Die so urdeutsche Unsitte des Trinkens wird nirgendswo besser beschrieben als bei dem Saufgelage in Hanau:

Ich sah einmal, daß diese Gäste die Trachten fraßen wie die Säue, darauf soffen wie die Kühe, sich dabei stellten wie die Esel, und alle endlich kotzten wie die Gerberhund. Den edlen Hochheimer, Bacharacher und Klingenberger gossen sie mit kübelmäßigen Gläsern in Magen hinunter, welche ihre Wirkungen gleich oben im Kopf verspüren ließen ... zuletzt verkehrte sich ihr Kampf in eine unflätige Sauferei. . . . . . . . . . . . . . . . . . .

Endlich setzt' es unten am Tisch ernstliche Streithändel, da warf man einander Gläser, Becher, Schüssel und Teller an die Köpfe und schlug nicht allein mit Fäusten, sondern auch mit Stühlen, Stuhlbeinen, Degen und allerhand Siebensachen drein, daß etlich der rote Saft über die Ohren lief. 11

Obwohl er wahrscheinlich kein Abstinenzler war, was für einen Schankwirt höchst ungewöhnlich wäre, bewies er doch eine erstaunliche Einsicht in die Schadwirkungen des Alkohols. So sagte er, daß "mehr durch Wein als durchs Schwert fallen",12 und auch die Grabinschrift für seinen verstorbenen Freund auf der Insel spricht für sich:

${ }^{10}$ Herbert Heckmann, "Grimmelshausen, oder die Leiden eines Volksschriftstellers," in W. Hinderer, Literarische Profile - Deutsche Dichter von Grimmelshausen bis Brecht (Königstein: Athenăum Verlag, 1982), 24

${ }^{11}$ Simplicissimus 1,32

12 ebda, Continuatio, 2 
$\mathrm{DaB}$ ich hier und nicht im Meer bin worden begraben, auch nicht in der Höll, macht, daß um mich gestritten haben drei Ding: das erste der wütende Ozean, das zweite der grausame Feind, der höllische Satan; Diesen entranne ich durch Gottes Hülf aus meinen Nöthen. Aber vom Palmwein, dem dritten, ließ ich mich tödten. ${ }^{13}$

$\mathrm{Zu}$ Geld scheint der ehemalige Schaffner ein etwas zwiespältiges Verhältnis gehabt zu haben, worüber im 7. Kapitel noch mehr zu sagen sein wird. Im Simplicissimus spielt es eine wichtige Rolle. Simplicius findet es immer wieder, meist ohne sein eigenes Zutun, aber immer gerade zur rechten Zeit, wenn er in finanzielle Nöte geraten ist. Er verschwendet es aber immer wieder, um sich herauszuputzen, um Freunden zu gefallen oder für Speise und Trank. Er läßt dabei eine ungute Einstellung, die zwischen Genußwunsch und Bekehrung schwankt, durchblicken. ${ }^{14}$ Das könnte durchaus des Autoren eigene Lage reflektieren, da er selber ja als Finanzbeamter der Schauenburger täglich mit dem Wert des Geldes konfrontiert wurde, andererseits wohl selten genug davon hatte, um sich und seiner großen Familie ein sorgenfreies Dasein zu sichern.

Die Geldgier der Menschen verhöhnte er trotzdem ironisch, als er beobachtete, daß Geld die Menschen fröhlicher als ein Edelstein machen könne, es fördere das Träumen und stärke Herz und Verstand. Besonders gut eigne es sich jedoch als "Schmiralia" vor Gericht. Die Negativseite hebe aber alle Vorzüge auf, denn es

${ }^{13} \underline{\text { Simplicissimus }}$ Continuatio, 22

${ }^{14}$ Martin Stern, "Geld und Geist bei Grimmelshausen," Daphnis 5, 2 - 4 (1976), 460 
raube die echten Freunde und den Seelenfrieden. ${ }^{15}$

Einen schweren Stand hatten bei ihm die Frauen. Obwohl das weibliche Geschlecht nur eine untergeordnete Rolle im Simplicissimus spielt und keine Charaktertiefe erfährt, werden die wenigen auftretenden weiblichen Figuren durchweg schablonenhaft negativ gezeigt. In seiner Einstellung den Frauen gegenüber folgt Grimmelshausen weitgehend der Konvention seiner Zeit. Während des 30jährigen Krieges war eine außerordentliche Verrohung im Verhältnis der Geschlechter zueinander eingetreten, die sich besonders in den niederen Kreisen, bei Tagelöhnern, Bauern und Soldaten, zu einer offenen Verachtung der Frau steigerte.16 Die Menschen waren moralisch und sittlich tief gesunken. Man genoß die unzüchtigsten Handlungen und fand Gefallen an den rohesten Beschreibungen. Die Liebe wurde zwar in den heroisch-galanten Romanen idealisiert, die Wirklichkeit sah aber das genaue Gegenteil dieser Einstellung, wobei die Frau die Hauptschuld zu tragen bekam. In ihr sah man alle menschlichen Laster und Fehler vereinigt. Sie wurde typischerweise als leichtfertig, verschwenderisch, boshaft und unkeusch charakterisiert. ${ }^{17}$

Dementsprechend wurden die Liebesabenteuer des jungen

${ }^{15}$ Simplicissimus III, 13

16R. Lochner, 81

${ }^{17}$ Siegfried Streller, Grimmelshausens simplicianische Schriften. Allegorie, Zahl und Wirklichkeitsdarstellung (Berlin: Rüttgen \& Loening, 1957), 246 
Simplicius von Grimmelshausen als geistig höchst unbefriedigend beschrieben. In Lippstadt, wo der junge Troßbube sexuell erwacht, treibt er es gleich mit sechs Mädchen: "Ich hatte gerade sechs, die mich liebten und ich sie hinwiederum, doch hatte keine mein Hertz gantz oder mich allein."18 Er muß heiraten, verläßt aber seine Frau sofort wieder und sieht sie im Verlauf der Geschichte nicht mehr wieder. Über Paris, wo er zum Prostituierten von vier adeligen Damen wird, kommt er in den Schwarzwald, wo er zum zweiten Mal heiratet, allerdings mit ebensowenig Glück. Die Ehe mit einer Trinkerin endet schon kurze Zeit später.

Die Frage drängt sich auf, ob der Autor die verächtlichen Beschreibungen der Frauen nur als Zeitzeichen wahrnahm, oder ob er diese Einstellung aus Überzeugung teilte. Das von ihm moralisch völlig verwerflich entwickelte Bild der Courasche scheint auf das letztere hinzudeuten. Andererseits bemerkt Gerhard Teuscher, daß der unabänderliche Niedergang der Courasche durchweg durch die Männer verursacht wurde, und Grimmelshausen daher eine eher fortschrittliche Differenzierung im Ansehen der Frau bekundet. ${ }^{19}$ Seine eigene Ehe mit Catharina Henninger, die ihm zehn Kinder bescherte, schien nicht sonderlich unglücklich gewesen zu sein. Zumindest verrät das wiederholte Auftauchen der "Meuder" im Simplicissimus, im Springinsfeld und im Ewigwährenden Calen-

${ }^{18}$ Simplicissimus $\mathrm{III}, 18$

${ }^{19}$ Gerhard Teuscher, "Fromme tugendhafte Frauen oder arglistiges Weibervolk?" in Jahrbuch für Internationale Germanistik, 16, 1 (1984), 96 
der, daß Grimmelshausen die Rolle der Mutter wohl zu schätzen gewußt hatte. ${ }^{20}$ Wenn er also auch die Gefühle seiner Zeit der Frau gegenüber deutlich darstellte und wahrscheinlich im Prinzip auch teilte, so besaß er doch genügend Übersicht, um die oftmals provokatorischen Handlungen des Mannes bloßzulegen.

Die weibliche Eitelkeit und modische Ausschweifungen verwarf er aber streng. Er ließ den Simplicius frech sagen: "Unser Aff trägt seinen Hintern bloß, diese Dame aber allbereit ihre Brüst."21 Auch das Tanzen verurteilte er als leichtfertiges Affenspiel, bei dem sich die Menschen nur zu leicht etwas an den Hals laden, was sie bald schon bereuen. So kommt das Bild des Renchener Bürgermeisters als das eines scharfen Kritikers des gesellschaftlichen und sittlilichen Niedergangs, den er als aufmerksamer Individualist rund um sich beobachtete, über die Zeit.

Von seiner zweifelhaften Fraueneinstellung schien auch der Familiensinn Grimmelshausens getrübt. Zwar notierte er das Verhältnis des jungen Simplicius zu seiner "Meuder" mit einiger Herzlichkeit, es wehte jedoch immer ein kräftiger Hauch Ironie mit in der Beschreibung. ${ }^{22}$ Auch kann die Beschreibung des warmen Verhältnisses zwischen Herzbruder und dessen Vater nicht über die grobe Vater-Sohn Einstellung des Autoren

${ }^{20}$ S. Streller, 247

${ }^{21}$ Simplicissimus II, 9

${ }^{22}$ ebda I, 3 
hinwegtäuschen.

Offenbar spielte der frühe Verlust seines eigenen Elternhauses, Grimmelshausen war erst fünf Jahre alt, als seine Mutter Gelnhausen verließ und er in die Obhut seines Großvaters kam, eine wichtige Rolle in seinem schwach entwickelten Familiensinn. Hinzu kam bei ihm der Argwohn zwischen den Geschlechtern und die absolute Ausrichtung auf die Vormachtstellung des Mannes in der Gesellschaft des 17. Jahrhunderts, die seine konventionelle Haltung bestärkte. Leider liegt seine eigene Rolle als Vater im Dunkel der Vergangenheit. Verschiedene Bemerkungen lassen jedoch darauf schließen, daß er sich seinen Kindern gegenüber bevorzugt in die seiner Zeit durchaus angemessenen Position des weisen, aber strengen Patriarchen gegeben hat. ${ }^{23}$ Es wäre aber sicherlich abwegig zu behaupten, daß der Renchener Bürgermeister ein engstirniger Pedant gewesen sei. Dagegen sprechen sein erstaunlicher Weitblick, seine Weltoffenheit und sein gesunder Humor. In seinem Verhältnis zum menschlichen Zusammenleben verriet er jedoch wenig Fortschrittliches.

Diese kühle, vielleicht sogar unsichere Vergeistigung der zwischenmenschlichen Kontakte kann bei der turbulenten Jugend des Dichters nicht überraschen. Möglicherweise war er sich seiner Benachteiligung durch die schwierige Kindheit und Jugendzeit selber bewußt, denn er bewies Einsicht in die Bedeutung eines geordneten Elternhauses, als er die Charakterweise des Halunken 
Olivier durch dessen disziplinlose Erziehung erklärte. ${ }^{24}$ Der lange Krieg hatte zweifellos großen Einfluß auf den heranwachsenden Jungen. Das rohe, ausschweifende und haltlose Leben der Kroaten, Hessen und Schweden, bei denen sich der junge Grimmelshausen abwechselnd befand, mußte einen tiefen Eindruck auf den 12jährigen gemacht haben. Die Erlebnisse seiner formativen Jahre machten ihn zu dem scharfen Beobachter und Menschenkenner, als der er sich in allen seinen Werken zu erkennen gab. ${ }^{25}$

Der Krieg gibt dann auch den Leitfaden in seinem Hauptwerk ab. In den Schilderungen der Kriegsabenteuer des jungen Simplicissimus zeichnete sich Grimmelshausen als ausgezeichneter Kenner des wüsten Soldatenlebens während des 30jährigen Krieges aus. Es ist auffallend, daß der Stoff dabei niemals den Gesichtskreis der einfachen Landsknechte durchbricht. Der Leser erfährt nichts von den großen Plänen und Strategien der Generalität, die Gründe für Truppenbewegungen und Schlachten bleiben dem Leser ebenso verborgen wie sie zweifellos ein undurchsichtiges Rätsel für die meisten Soldaten jener Zeit waren, und wie sie es auch heute oft immer noch sind.

Als Schauenburger Schaffner spielte er die Vermittlerrolle zwischen den Aristokraten, in deren Dienst er stand, und den untergebenen Bauern des westlichen Schwarzwaldes, denen es zum Ende des 30jährigen Krieges sehr schlecht ging. Er erlebte täglich

${ }^{24}$ Simplicissimus IV, 18

25 R. Lochner, 175 
die große Armut der Menschen um ihn her, aber auch an seiner eigenen Person. Es geht aus fast allen Forschungsarbeiten hervor, $\mathrm{da} ß$ der Dichter mit großen finanziellen Schwierigkeiten zu kämpfen hatte. So ist es verständlich, wenn er die Verhältnisse seiner Zeit bevorzugt aus der Sicht des armen Volkes beschrieb. 26

Die Vorliebe des Renchener Schultheiß' für die einfachen Stände spricht aus allen seinen Büchern. Der Adel spielt nur eine periphere Rolle, unerreichtbar und unangefochten dominierend. In farbenfrohen, lebendigen Bildern führte der Dichter die verschiedenen Typen seiner Erlebniswelt vor, Dragoner, Musketiere, die Marodeure, die guten und schlechten Zeitgenossen als Herzbruder und Olivier, und das Beispiel der starken, aber einfältigen und unmündigen Soldatenseele in der Person des Springinsfeld, der wohl exemplarisch für die Mehrzahl der armseligen Söldner des Krieges steht.

Als er den Simplicissimus schrieb, lag die Soldatenzeit Grimmelshausens bereits fast zwanzig Jahre zurück, wobei aber als sicher angenommen wird, daß er schon während seiner Offenburger Zeit mit Aufzeichnungen seiner Beobachtungen begonnen hatte. Dieser Abstand, sowie die neuen Lebenskreise als Schaffner, Wirt und verantwortungsvolles Familienoberhaupt, schärfte und erweiterte seine Urteilskraft. So verdammte er das Soldatenleben ebenso wenig wie er es verherrlichte. Seine grundsätzliche Einstellung zum

${ }^{26}$ Renate Brie, Die sozialen Ideen Grimmelshausens (Lübeck: Matthiesen Verlag, 1938 \& 1967), 63 
Krieg und $\mathrm{zu}$ dem Schicksal der Betroffenen machte er in seiner berühmten Allegorie des Ständebaumes deutlich.27 In diesem treffenden Bild beschrieb er die sozialen Mißstände und Spannungen zwischen den Klassen und Ständen der Barockgesellschaft. In der differenzierten Beschreibung dieser brutalen Epoche der deutschen Geschichte liegt Grimmelshausens Stärke und Genialität.

Wie war es dem Schwarzwaldautoren möglich, eine so treffende Metapher in einer Zeit zu entwickeln, in der Unmündigkeit und Autoritätsdenken zum täglichen Leben der unteren Stände gehörte? Die Frage nach der Bildung des ehemaligen Gelnhausener Lateinschülers gehört zu den großen Streitfragen der Grimmelshausenforschung.

Es ist jedem Interessierten klar, daß der Stoff der simplicianischen Schriften, besonders des Simplicissimus, eine überdurchschnittliche Fülle von Fakten, Sagen und barockem Allgemeinwissen, in wenn auch manchmal etwas kunterbunter Reihenfolge, zur Schau stellt. Wie konnte ein Kleinbürger des 17. Jahrhunderts, noch dazu aus eher armseligen Verhältnissen, zu einem so breitgefächerten Wissen kommen? Es mutet umso erstaunlicher an, wenn man die formale Schulbildung von höchstens 6 bis 7 Jahre, von 1628 bis 1634, bedenkt. J. H. Scholte glaubt deswegen auch nicht an ein besonders tiefes Eindringen des jungen Grimmelshausen in gelehrte Materien. Er vermutet in dem jungen Gelnhausener lediglich 
Grundkenntnisse in Lesen, Schreiben, Rechnen, Religion und Latein, als dieser 1634 durch die Zerstörung seiner Heimatstadt gezwungen wurde, seine Schulausbildung abzubrechen.28 Richard Alewyn spricht Grimmelshausen fast jede Schulbildung ab und vermutet einen reinen Autodidakt. ${ }^{29}$

Der Renchener Bürgermeister gab selber zu, seinem Narren Simplicius sehr ähnlich gewesen zu sein, wie er im Vorwort des Satyrischen Pilgrim schrieb:

Man weiß ja wohl, daß er selbst nichts studirt, gelernt noch erfahren, sondern er kaum das $A B C$ begriffen hat in Krieg kommen im zehnjährigen Alter ein rotziger Musquetier worden, auch allwo in demselben liederlichen Leben ohne gute disciplin und Unterweisungen wie ein anderer grober Schlingel, unwissender Esel, Ignorant und Idiot, Bernheuterisch aufgewachsen ist.

Andererseits vermutet Günther Weydt auf Grund einer überlieferten Schulordnung der Gelnhausener Lateinschule von 1568, daß der Junge eine wesentlich intensivere Schulbildung genossen hat. 30 Er geht sogar so weit zu behaupten, daß Grimmelshausen Homer gelesen haben muß, weil die Handlung des Simplicissimus in vieler Hinsicht mit der Odysee der griechischen Antike übereinstimmt. ${ }^{31}$

28J.H. Scholte, 132

${ }^{29}$ Richard Alewyn, "Grimmelshausen Probleme," in G. Weydt, Der Simplicissimusdichter, 398

${ }^{30} \mathrm{G}$. Weydt, Nachahmung, 27 \& Anhang

${ }^{31}$ G. Weydt, "Grimmelshausens Bildung." Alte und Neue Kontroversen, Akten des VII. Germanisten Kongresses, Göttingen, 1985 (Tübingen: Max Niemeyer Verlag, 1986), 174 
Einwandfrei scheint festzustehen, daß der Dichter von seiner Jugend an ein lesewütiger Mensch war. Im Ewigwährenden Calender legte er Simplicius die durchaus autobiographisch anzusehenden Worte in den Mund: "Liebe Mutter, besser um Bücher das Geld zu vernarren als verspielt; ich hab doch sonst kein Freund in der Welt." Und im Simplicissimus findet sich noch ein Hinweis, wie er seine freie Zeit ausfüllte: "Wann ich aber wegen üblen Wetters in Wäldern und Feldern nicht herumb konnte schwermen, so lase ich allerhand Bücher, die mir des Closters Verwalter leyhte." 32

Die Tatsache, daß Grimmelshausen als Schreiber in die Sekretariatsstube des Schauenburger Regiments aufgenommen wurde, spricht ebenfalls für eine relativ gute Ausbildung. Dort traf er dann auf den Magister Johannes Witsch, der von der Universität Freiburg kam und in dem jungen Schreiber sicherlich einen wissensdurstigen Schüler vorfand. Auf der Schreibstube wurde wahrscheinlich der Grundstein für die imponierenden Literaturkenntnisse Grimmelshausens gelegt.

Zwar glaubt Julius Petersen, daß der Simplicissimusdichter hauptsächlich die volkstümliche deutsche Literatur des 16. Jahrhunderts für seine Werke zu Rate zog. 33 Vieles spricht hingegen dafür, daß er die gesamte europäische Barock- und Renaissancedichtung verwendete. Ganz sicher verdankte er viel dem 1619 in

${ }^{32}$ Simplicissimus II, 29

33J. Petersen, 64 
deutscher Übersetzung erschienenen Nachschlagewerk Piazza Universale des Italieners Tommaso Garzoni, aus dem sich häufig Passagen in fast allen Schriften Grimmelshausens nachweisen lassen.

Günther Weydt veröffentlichte 1968 eine beeindruckende Liste von 90 Büchern, von Albertinus' Gusman von Alfarache bis zu den Volksbüchern Faust und Eulenspiegel, die der Simplicissimusautor nachweislich für seine Arbeiten verwendet hat.34 Dabei kann man wohl noch nicht von einer endguiltigen Sammlung sprechen. Diese große Anzahl machte den Schwarzwälder Schultheiß zu einem so hochbelesenen Mann, daß er im barocken Deutschland durchaus zu den Gebildeten seiner Zeit zu rechnen war. Von einer "krausen Gelehrsamkeit", wie Alewyn sie bei Grimmelshausen zu erkennen glaubt, kann nur aus der Sicht des erheblich fortgeschritteneren 20. Jahrhunderts gesprochen werden.

Wie konnte Grimmelshausen, der ja schon aus finanziellen Gründen unmöglich so viele Bücher selber besessen haben konnte, einen solch breiten Einblick in die Literatur seiner Zeit nehmen? Weydt vermutet, daß er Kontakte zu den großen Bibliotheken unterhalten hatte. 35 Es wird angenommen, daß Grimmelshausen als Vogt auf der Ullenburg Zugang zu der reichhaltigen Büchersammlung seines Brotherrn Dr. Küffer hatte, der Mitglied der Straßburger Sprachgesellschaft "Von der Tannen" war. Diese von Adligen, Gelehrten und Dichtern besetzten Vereinigungen, deren berühmteste die

${ }^{34}$ G. Weydt, Nachahmung, Anhang

${ }^{35} \mathrm{G}$. Weydt, "Bildung," 170 
"Fruchtbringende Gesellschaft" war, hatten es sich zur Aufgabe gemacht, die deutsche Sprache von Fremdwörtern zu säubern und sie den anderen mitteleuropäischen Sprachen ebenbürtig zu machen. Obwohl Grimmelshausen den Reformbestrebungen der Sprachgesellschaften im Prinzip folgte, schaute er doch mit unverhohlenem Spott auf die übertriebenen Aktivitäten dieser Vereinigungen. So höhnte er im Ewigwährenden Calender offen:

Er sah bei den Schweizern unterschiedlich Esel und Maultier mit Zitronen, Lemonen, Pommeranzen und sonst allerhand Waren aus Italia über das Gebirg kommen; da sagte er zum Herzbruder, schauet um Gotteswillen, das ist der Italiener fruchtbringende Gesellschaft.

Die Dichtkunst sollte seiner Meinung nach weder den Spuren alter Poeten folgen, noch in selbstherrlicher Sprachpflege untergehen. 36 $\mathrm{DaB}$ der Simplicissimusdichter sich gegen die trendbewußten $\mathrm{Be}$ mühungen seiner Zeit stellte, wirft ein bezeichnendes Licht auf seine Einstellung. Er fühlte sich kompetent genug, um gegen die anerkannten Literaturkreise Deutschlands forsch anzugehen, und er wagte dank seiner Erfahrung, die verschnörkelte Dichtung des 17. Jahrhunderts zu verlachen. Er bewies damit, bewußt oder rein instinktiv, eine sprachliche Sicherheit, wie sie nur wenige seiner zeitgenössischen Schriftstellerkollegen offenbarten.

Mit seinen scharfen Angriffen gegen die "Sprachverderber" manövrierte sich der Simplicissimusdichter gegenüber den ge-

36Paul Böckmann, "Die Abwendung vom Elegantaideal in Grimmelshausens Simplicissimus," in Formengeschichte der deutschen Dichtung Bd. 1 (Hamburg: Hoffmann \& Campe,1967), 448 
lehrten Kreisen natürlich ins Abseits. So schrieb Quirin Moscherosch, ein Bruder des Satirikers Johann Michael Moscheroschs und selber Mitglied des Nürnberger "Hirten- und Blumenorden", 1673:

Es hat der beruffene Simplicissimus, sonsten mein Nachbar, und ein geringer Dorfschultes, aber ein DauB EB (ein nichtswürdiger Mensch) u. homo Satyricus in folio bey H. Felßeckern vor weynachten ein Tractätlein trucken lassen, dessen Titel des Teutschen Michels Sprachgepräng, nach art des Malers Farben gemäng, darinnen er die Teutschen Sprach-Helden recht Satyrisch anzäpfet; möchte wol wünschen, wann Ihm einer nur mit 1 paar bögen das Maul stopfte, wanns mein Amt zuliesse, wolte ichs nicht underlassen. Halte aber, er werde in ein wespennest gestochen haben, die sich schon an ihm rechen werden . . .37

Die Stellung im literarischen Niemandsland schien Grimmelshausen nicht sonderlich gestört zu haben. Er fühlte sich von Natur her mit den ungezwungenen, derben Bauern und Handwerkständen verbunden, in deren Sprache er hauptsächlich schrieb.

Ein ähnlicher, für ihn nicht untypischer Widerspruchsgeist zeigte sich in Grimmelshausens politischer Einstellung. Seine beruflichen Kontakte als Schaffner gaben ihm die Gelegenheit, mit dem Adel ebenso wie mit den untertänigen Bauern zusammenzukommen. Dem Simplicissimusautoren konnten die krassen Unterschiede und Spannungen zwischen den Ständen nicht entgehen, wie das schon erwähnte Beispiel des Ständebaumes aufgezeigt hat. Sein wohl deutlichstes politisches Bekenntnis findet sich in der Jupiter-Episode. 38

${ }^{37}$ Ch. Stoll, 49

${ }^{38}$ Simplicissimus III, 4 \& 5 
Hier malte Grimmelshausen das utopische Bild eines zentralistisch regierten Staates, in dem die klügsten und gelehrtesten Männer mit absolutistischer Macht über eine standeslose und gleichberechtigte Bürgerschaft regieren, und in dem aristokratische Privilegien unbeachtet bleiben. Mit der Absetzung der deutschen Kleinfürsten, die den Zusammenhalt des deutschen Weltreiches gefährden, und der Einführung eines Parlamentes bewies der Renchener Bürgermeister, daß er politisch seiner Zeit weit voraus war.

Die von ihm im Ratio Status und in der Mummelsee-Szene 39 entworfene Regierungs- und Sozialordnung verband er jedoch noch fest an die moralisch-theologischen Prinzipien des Spätmittelalters. 40 Die Staatsordnung bezog ihre Legitimität aus dem göttlichen Willen und aus moralischem Recht. Das Sylphenreich im Mummelsee zeigt, daß eine konfliktfreie Gesellschaft nur solchen Lebewesen vorbehalten bleibt, die nicht sündigen können, und die keine Alternative zu richtigem Verhalten haben. Grimmelshausen erkannte klar, daß diese Sozialordnung für die Menschen ihrer Sündhaftigkeit wegen eine Utopie bleiben mußte.

Den Gedankengang verfolgte er noch einmal in seiner Beschreibung der Wiedertäufer in Ungarn ${ }^{41}$, denen die Möglichkeit zur Sünde

${ }^{39}$ Simplicissimus V, 13

40Dieter Breuer, "Grimmelshausens politische Argumentation," Daphnis 5, 2 - 4 (1976), 303

${ }^{41}$ Simplicissimus V, 19 
durch strengste Disziplin genommen wurde. Aber die Undurchführbarkeit dieser Lebensweise als Allgemeinprinzip spricht aus den Worten, mit denen er die Szene abschloß: "Aber mein Knan prophezeite mir stracks, daß ich wohl nimmermehr solche Bursch zusammenbringen würde." Somit zeichnete er sich als ein realistischer politischer Denker aus.

Die politischen und moralischen Anschauungen Grimmelshausens wurzelten tief in seiner religiösen Überzeugung. Er war zweifellos religiös, wie die Religion für den Menschen des Barockzeitalters eine tragende Funktion ausübte, obwohl gerade der religiöse $\mathrm{Be}$ reich die Menschen in starke Widersprüche und Zweifel verstrickte. Mittelalterliche Überlieferungen, wie christliche Askese und Glauben an die menschliche Unzulänglichkeit, standen einer neuen Gedankenwelt gegenüber, die materielle Sicherheit und Verlangen nach Wissen und Genuß hervorhob. Ein empfindsamer Mensch, wie der Dichter der simplicianischen Schriften zweifellos war, mußte durch diese Aufsplitterung der alten Wertvorstellungen vor schwer zu lösende persönliche Probleme gestellt werden.

Grimmelshausen war protestantisch aufgewachsen, da Gelnhausen stark unter lutherischem Einfluß stand. Es ist nicht bekannt, wann und warum er zum katholischen Glauben übertrat, aber er heiratete 1649 als Katholik. Es läßt sich vermuten, daß es der ständige Umgang mit kaiserlichen Soldaten in der Garnisonstadt Offenburg, oder auch ein Zugeständnis an die einflußreiche Familie seiner Frau war. Er ist allerdings auf keinen Fall ein engstirniger 
Religionsfanatiker gewesen. Eher hielt er es mit dem Spruch Friedrich Logaus, den er im Teutschen Michel zitierte:

Luthrisch, Päpstisch und Calvinisch, diese Glauben sind drei, sind vorhanden, doch ist Zweifel, wo das Christentum denn sei. 42

Er las mit Sicherheit die Schriften des Münchener Hofsekretärs Aegidius Albertinus, der neben gegenreformatorischer Propaganda auch den Schelmenroman Gusman de Alfarache aus dem Spanischen übersetzt hatte. Trotzdem hielt Grimmelshausen an gewissen protestantischen Anschauungen fest, wie Scholte beobachtet. ${ }^{43}$ Seine Meinung über gottbefohlene Einsamkeit und christliche Askese verkündete der Verfasser des Simplicissimus mit feiner Ironie in der Überschrift des letzten Kapitels seines Einsiedlerlebens am Anfang des Buches: "Vermerckt eine schöne Art zu sterben und sich mit geringen Unkosten begraben zu lassen."44

Auch Simplicius' Wallfahrt durch die Schweiz mit Herzbruder ist bezeichnend für die Diesseitigkeit des Autoren. Simplicius ist zwar damit einverstanden, seine Schuhe mit Erbsen zu belegen, aber er kocht sie vorher heimlich. 45 Welch ein urwüchsiger Humor spricht aus dieser Idee!

Grimmelshausen erkennt die religiöse Weltentsagung als Ver-

42J. Petersen, 68

43J. H. Scholte, 183

${ }^{44}$ Simplicissimus I, 12

45 ebda V, 1 
wirklichung des christlichen Glaubens nicht an.46 Statt dessen sieht der Dichter in der Selbsterkenntnis und der richtigen, d.h. moralisch-ethischen, Art zu leben die Grundlage für ein christliches Dasein, daß von dem Menschen als Ebenbild Gottes verlangt wird. In dieser Rolle kann der Mensch seiner Gemeinschaft dienen. In der bewußten Durchbrechung der engen konfessionellen Grenzen zeigte Grimmelshausen eine fortschrittliche Glaubenseinstellung, die selbst heute noch nicht bei allen Westeuropäern vorherrscht

\section{ZUSAMMENFASSUNG}

Welches Bild ergeben die verschiedenen Charakterfärbungen und Eigenschaftsmuster nun? Am wohl deutlichsten und von niemandem angezweifelt ist Grimmelshausens überdurchschnittlich scharfe Beobachtungsgabe, die ein Beweis für einen höchst lebendigen Geist ist. Der Renchener Schultheiß stand mitten im Leben und beobachtete hellwach seine Umgebung, wobei er sich nicht nur seiner Augen bediente. Hellhörig nahm er die verschiedenen Mundarten auf, die er während seiner Soldatenzeit hörte, und gab sie getreu in seinen Schriften wieder.

Wahrscheinlich war er kein Grübler, aber er sann doch über das Erfahrene nach und war bestrebt, es in sein bestehendes Weltbild

46Italo Battafarano, "Grimmelshausens Kritik an der Ideologie seiner Zeit," Daphnis 5, 2 - 4 (1976), 300 
zu integrieren. Als Konsequenz erweiterte sich sein Horizont im Laufe seines Lebens ständig, wobei seine Leselust ihm immer neue Bereiche eröffnet haben dürfte.

In vieler Hinsicht, namentlich in sozialen, politischen und religiösen Aspekten, war er seiner Zeit weit voraus. Er fühlte sich zeit seines Lebens dem einfachen Volk zugetan, obwohl er sich, vermutlich aus wirtschaftlichen Gründen, den höheren Ständen zu nähern versuchte. Man kann in ihm die wachsende Einsicht vermuten, daß der einzelne eine große Verantwortung der Gesellschaft gegenüber trägt. Er war sich sicherlich seiner Verantwortung gegenüber seiner Gemeinde, aber auch als Schriftsteller seinen Lesern gegenüber bewuBt. 47 Seine Werke verraten einen hohen moralischen und sittlichen Standard, den zu offenbaren er nicht müde wird.

Eng mit seinem moralisch-ethischen Empfinden ist seine religiöse Einstellung verbunden. Auch hier überwog bei ihm der Realist, obwohl er vermutlich auch seinem Zeitgeist entsprechend an Hexenund Dämonenglauben festhielt. Ein religiöser Fanatiker war er keinesfalls, aber er trat als ein Verfechter des richtigen Lebens hervor. Dieses Dasein bestand für ihn in einer strikten Ablehnung ausschweifender Vergnügungen, wie Trinken, Tanzen oder Rauchen. Sexuelle Leichtlebigkeit lehnte er ebenso ab wie ein künstliches Absondern von der Gesellschaft der Mitmenschen.

Die Anforderungen seiner 11köpfigen Familie und sein persön-

${ }^{47}$ Gisela Herbst, Die Entwicklung des Grimmelshausenbildes in der Wissenschaftlichen Literatur (Bonn: H. Bouvier u. Co. Verlag, 1957), 9 
licher Ehrgeiz zwangen ihn zu ständiger Betriebsamkeit. In der Beschäftigungslosigkeit sah er eine Ursache vieler Übel. Seine Einstellung zur Arbeit im Dienst der Gemeinschaft wird von Renate Brie sogar "sozialistisch" genannt, 48 obwohl dieser Ausdruck vielleicht etwas übertrieben anmutet. Seinem Allgemeinbewußtsein stand ein starkes Selbstvertrauen zur Seite, daß mit einem energischen Selbstbehauptungswillen gepaart ihm bei seinem literarischen Zwist mit den gelehrten Kreisen seiner Zeit half.

Trotz dieses radikal aussehenden Zuges war Grimmelshausen alles andere als ein erneuerungswütiger Revolutionär. Er stand der Tradition des 16. Jahrhunderts in christlicher Motivation und konservativ gefestigter Lebensanschauung näher, als den am geistigen Horizont schimmernden Veränderungen der Aufklärung. ${ }^{9}$ Obwohl er ein erstaunliches $\mathrm{Ma} B$ an Phantasie und Vorstellungskraft besaß, hielt er doch an dem überlieferten Weltbild fest.

Dieses Dilemma kam bei seiner Einstellung zu Geld und Reichtum am deutlichsten zum Vorschein. Die christlich-mittelalterliche Doktrin verlangte absoluten Verzicht auf irdische Genüße, wobei das Geld als die schlimmste Versuchung schlechthin galt. Als armer Musketier, Schreiber und Schaffner sah Grimmelshausen aber die Annehmlichkeiten und die Notwendigkeit des Geldes täglich vor Augen. Trotzdem passte Reichtum als Teufelswerk immer noch besser in seine Weltanschauung als eine von Menschen geschaffene und

${ }^{48}$ R. Brie, 97

49J. H. Scholte, 89 
gelenkte Wirtschaft. 51

Und doch ist sein Entsagungswille nicht aus vollstem Herzen gekommen. Aus seinem Werk scheint er als ein letztendlich weltoffener, frohgemuter und ehrlicher Mensch zu sprechen, dessen humorvoll-verschmitzten Worte "Es hat mir so wollen behagen, mit Lachen die Wahrheit zu sagen" deutliche Auskunft über seine reife Gesamteinstellung geben.52

51M. Stern, 436

${ }^{52} \mathrm{R}$. Lochner, 178 


\section{KAPITEL}

\section{GRIMMELSHAUSENS ABSICHT BEI DER SCHAFFUNG SEINES HAUPTWERKES}

Die zahlreichen Auslegungsmöglichkeiten, zu denen sich der Simplicissimus hergibt und die im 5. Kapitel behandelt wurden, machen die Vielseitigkeit und Komplexität dieses ersten neuhochdeutschen Romans deutlich. Eine abschließende Interpretation wird vermutlich in absehbarer Zeit nicht möglich sein, weil das Werk wohl in seiner Gesamtheit beurteilt werden muß, wie Clemens Lugowski schon vermutet hat. ${ }^{1}$

Es handelt sich, so Lugowski, um eine literarische Großform, die sich aus verschiedenen Sonderformen zusammensetzt. Zwar ist es möglich, einzelne Sonderformen herauszufiltrieren, wie zum Beispiel den Typus der Schwankerzählung, der Litanei, oder der heroisch-galanten Wiedertreffen-Erzählung, aber die Verknüpfungspunkte sind im Simplicissimus so miteinander verwachsen, daß eine Aufschlüßelung fast unmöglich erscheint. Den Roman als Großform ohne harmonisierenden Zusammenhang zu erläutern, ist jedoch ebenfalls unbefriedigend und ruft darüber hinaus ständig neue Deutungsversuche hervor.

\footnotetext{
${ }^{1}$ C. Lugowski, 171
} 
Vielleicht könnte die Beantwortung der Frage nach der Absicht Grimmelshausens bei der Schaffung seines Werkes neue Einsichten bringen. Welche Ziele verfolgte der Renchener Bürgermeister mit dem Entwurf seines Romanhelden Simplicius? Leider verblieben keine Hinweise, die diese Frage unmittelbar beantworten könnten. Die wahre Intention des fleißigen Autoren kann also nicht mit letzter Sicherheit bewiesen werden. Es können jedoch Vermutungen angestellt werden, die sich auf die Rekonstruktion der damaligen Verhältnisse stützen.

Im Laufe dieser Arbeit wurde eine solche Wiederbelebung der Verhältnisse, wie sie auf den Dichter eingewirkt haben, angestrebt. Nun soll aus der Abwägung der bekannten Einflüße die Frage nach den Gründen der Entstehung des Simplicissimus synthetisiert werden.

Die meisten Grimmelshausenforscher scheuen sich, dieses Problem auch nur andeutungsweise $\mathrm{zu}$ behandeln, wohl wegen des hohen Unsicherheitsfaktors der Hypothese. Trotzdem läßt manch einer der Fachexperten eine Vermutung hier oder da aufleuchten. So vermutet J. H. Scholte, daß Grimmelshausen nach seiner Anstellung auf der Ullenburg aus finanzieller Not zur Feder griff. ${ }^{2}$ Der ehemalige Regimentsschreiber habe sich, arbeitslos, vor das Problem gestellt gesehen, eine vielköpfige Familie in schwieriger Zeit zu ernähren.

Es ist bekannt, daß er im Anschluß an seine Zeit auf der Ullen- 
burg auf seinem Besitz in Gaisbach eine Dorfschenke eröffnete. In den beiden folgenden Jahren wird er sich neben seinen Gastwirtspflichten auch an seine Schreibkenntnisse aus der Offenburger Militärzeit erinnert haben. Scholte sieht von Anfang an die Schaffung eines planmäßigen, ausbaufähigen Literaturwerkes in Grimmelshausens Absicht. ${ }^{3}$ Tatsächlich ist erwiesen, daß der Gaisbacher Gastwirt in finanzieller Not war. In einem Gutachten des Obervogtes und Amtsschaffners von Oberkirch wird er noch im Jahre 1667, dem Jahr der Simplicissimus-Veröffentlichung, als "unvermöglich" registriert. ${ }^{4}$ Scholte kann also durchaus berechtigt die Situation Grimmelshausens als verzweifelt ansehen, als der sich daran setzte, den Simplicissimus zu schreiben.

$\mathrm{Zu}$ ganz anderer Ansicht hingegen kommt Manfred Koschlig. Er glaubt, daß Grimmelshausen nicht geschrieben hat, weil ihm finanziell das Wasser bis zum Hals stand, sondern eher, weil es Deutschland politisch, sozial und religiös schlecht ging.5 Koschlig sieht in allen Werken des Schwarzwälder Dichters die Grundabsicht manifestiert, "Bescheid zu geben im Sinne des Ratens und Warnens".

Ferdinand von Ingen glaubt ebenfalls an die tiefe Besorgnis des Simplicissimus-Autoren, der die Menschen für unfähig hielt,

3J. H. Scholte, 173

${ }^{4}$ H. D. Gebauer, 405

${ }^{5}$ Manfred Koschlig, "Das Mythos vom Bauernpoeten Grimmelshausen" in Das Ingenium, 157 
auch nach langer Kriegszeit einen Frieden durch Bekehrung einzuhalten. 6 Der Schriftsteller habe es als seine Aufgabe angesehen, seine Mitmenschen vor dem kommenden Weltgericht zu warnen und zur Besserung zu mahnen. Wie lassen sich nun die oppositionellen Meinungen Koschligs, von Ingens und Scholtes miteinander verbinden?

Um zu einem wahrscheinlichen Bild der Lage zu kommen, muß man nicht nur die äußeren Gegebenheiten begutachten, sondern auch die Persönlichkeit des eben arbeitslos gewordenen Schaffners und Burgvogtes berücksichtigen. Er war, wie im 5. Kapitel bereits ausführlich erklärt worden ist, ein gewissenhafter, von hoher moralisch-ethischer Sensibilität geleiteter Mensch. Gleichzeitig kann er als ein selbstsicherer, energischer und arbeitsamer Mann betrachtet werden, dem Beschäftigungslosigkeit ein Greuel war. Für solch einen Menschen wäre es undenkbar, seine von ihm abhängige Familie durch resignierte Müßigkeit in großer Not zu belassen. Es ist unwahrscheinlich, daß er die Burgvogtanstellung wegen des Planes aufgegeben hat, Gastwirt und Schriftsteller zu werden. Eher ist zu vermuten, daß die Entscheidung, den "Silbernen Stern" in Gaisbach zu eröffnen, eine Notlösung war, die er aber schnell tatkräftig zu einer Tugend umpolte. Wahrscheinlich war der Gedanke, Bücher zu verfassen, während seiner Ullenburgzeit gereift, wo er ja Kontakte mit den literarischen Kreisen Dr. Küffers in Straßburg bekommen

6Ferdinand von Ingen, "Krieg und Frieden bei Grimmelshausen," in Etudes Germaniques (Jan-Mar 1991), 50 
hatte.

Grimmelshausen war, wie Koschlig feststellt, ein Außenseiter in seiner Welt. ${ }^{7}$ Als solcher konnte er aber seine Umgebung, die Bauern ebenso wie die adelige Gesellschaft, treffend beurteilen, wobei ihm seine ausgezeichnete Beobachtungsgabe behilflich war. Als seine Amtszeit auf der Ullenburg, aus welchem Grund auch immer, zu Ende ging, griff er die Gelegenheit beim Schopfe, seine Beobachtungen und wohlgeformten Überzeugungen schreibend $\mathrm{zu}$ verkünden. Als Wirt, dem sicher von den älteren seiner Kinder kräftig zur Hand gegangen wurde, fand er nun Zeit, sich seiner Berufung ausgiebig zu widmen.

Die überdurchschnittlich große Anzahl seiner Veröffentlichungen, die während seiner Gastwirtzeit und während der ersten Jahre als Bürgermeister entstanden sind, zeigen, daß er zwischen 1665 und 1670 unabläßig geschrieben haben muß. Getrieben wurde er dabei sicher von einem tief empfundenen Pflichtgefühl, die Unsitten und Mißstände seiner Zeit aufzuzeigen. Seine eigenen Kriegserlebnisse lieferten dabei den willkommenen Hintergrund. Verschiedentlich wies er ausdrücklich auf diese Berufung hin, seine Mitmenschen aufklären zu wollen, ${ }^{8}$ wobei er es an unbequemen Entdeckungen nicht fehlen ließ. Er war aber realistisch und vorsichtig genug, seine harsche Kritik besonders an der aristokratischen Welt sati-

${ }^{7}$ M. Koschlig, "Das Mythos," 121

${ }^{8}$ Als Beispiel, siehe Simplicissimus I, 9 und IV, 13 
risch $\mathrm{zu}$ verkleiden und seine Werke nicht unter seinem richtigen Namen zu veröffentlichen, denn er wußte, daß er auch weiterhin auf das Wohlwollen des Adels in seiner Umgebung angewiesen sein würde. Daher verbarg er sich hinter dem Anagramm "German Schleifheim von Sulsfort". Kurz vor der Veröffentlichung des Simplicissimus hatte sich der Gaisbacher Wirt um die Schultheißenstelle beim Bischof von Straßburg beworben, und diese Position wollte er sich sicherlich nicht durch seine schriftlichen Werke verderben.

Seit Hans Dieter Gebauers Untersuchungen über die potentielle Leserschaft während des 17. Jahrhunderts steht einwandfrei fest, daß Grimmelshausen seine Werke, besonders aber den Simplicissimus, für die gehobene Klasse geschrieben hat. Er wußte, daß Bauern und Tagelöhner sein vielseitiges Hauptwerk höchstens durch Zufall in die Hände bekommen würden. ${ }^{9}$ Der Preis des Simplicissimus, die Lesefähigkeit der Menschen und auch die Muße, ein so aufwendiges Buch zu lesen, schloß das niedere Volk als Zielgruppe aus. Es waren die Aristokraten und die Menschen des gehobenen Mittelstandes, die Grimmelshausen ansprechen wollte, um ihnen die Verkommenheit ihrer Zeit ans Herz zu legen. Der Autor spürte, daß er seine Zeitgenossen auf die von ihm erkannten sozialen, sittlichen und wirtschaftlich-politischen Veränderungen im Leben seiner Zeit aufmerksam machen wollte. Es folgert daher

${ }^{9}$ H. D. Gebauer, 409 
mit einiger Sicherheit, daß der Simplicissimus hauptsächlich aus moralisch-ethischem Sendungsbewußtsein entstanden ist.

Der Erfolg seines Werkes kam für ihn sicherlich völlig überraschend. Es ist kaum anzunehmen, daß er, wie Scholte vermutet, hauptsächlich Geschäftsmann war. ${ }^{10}$ Diese Vermutung ist bei dem beobachteten zwiespältigen Verhältnis des ehemaligen Schauenburger Schaffners zu Geld und Kapital kaum aufrecht zu erhalten. $\mathrm{Zu}$ fest stand Grimmelshausen in der Überzeugung des 16. Jahrhunderts, das von der Sündhaftigkeit des Geldes überzeugt war. Vielmehr ist anzunehmen, daß die verschiedenen Veränderungen am Simplicissimus (z.B. die Titelkupfer) durch den Verleger Felßecker mehr oder weniger eigenhändig vorgenommen worden sind, um das Buch immer lukrativer zu gestalten. Der Renchener Autor stimmte den kapitalistischen Anliegen seines Verlegers aber zu, denn auch ihm war das Bewußtsein von der Macht des Geldes nicht entgangen. Und sicherlich sah er mit dem gesteigerten Einkommen die wirtschaftliche Sicherheit seiner Familie gewährleistet, für die er letztendlich allein verantwortlich war.

Natürlich lassen sich noch andere Motive denken, die Grimmelshausen bewegt haben könnten, seinen Simplicissimus zu schreiben. Vielleicht sollte es eine Religionssatyre werden, wie Scholte auf Grund der Wahl des Verlagsortes ${ }^{11}$ vorschlägt. Die liberale Ein-

10J.H. Scholte, "Die Restaurierung des Simplicissimus," in G. Weydt, Der Simplicissimusdichter, 430

${ }^{11}$ siehe Kapitel 3 
stellung des Schwarzwälder Bürgermeisters den Konfessionen gegenüber unterstützt diese Vermutung. Aber das Werk ist doch zu komplex und beleuchtet zu viele Schichten der Barockgesellschaft, um diese These als alleinige Triebfeder gelten zu lassen. Eher ist es wahrscheinlich, daß der Schriftsteller die Konfessionsstreitereien als eines der Mißstände seiner Zeit mit in den zu behandelnden Stoffkatalog einbezog.

Möglich, aber kaum glaubwürdig, ist der Gedanke, im Simplicissimus eine Aufarbeitung des Lebens Grimmelshausens zu sehen, die der Autor aus einer Lebenskrise heraus vornehmen wollte. Zwar waren die Jahre zwischen 1665 und 1667 keine ruhige und ausgeglichene Zeit für den stellungslosen Schaffner, aber seine Selbssicherheit und sein moralisch gefestigtes Weltbild widerlegen die Vorstellung eines nach Halt und Lebenssinn Suchenden.

Der geschickt angelegte Aufbau des Romans zeigt deutlich, daß es dem Dichter um eine Botschaft ging, die er ausstrahlen wollte. Er war durch seinen Beruf ein an Organisation und Einteilung gewöhnter Mensch, dem systematisches Arbeiten nicht fremd war. Die von ihm zu führenden Akten und Bücher während seiner Schaffnerzeit, aber auch schon während seiner Zeit als Regimentssekretär, haben in ihm zweifellos einen Sinn für Ordnung, Sorgfalt und Planmäßigkeit wachsen lassen. Diese Charakteristiken benutzte er, um sein unbestreitbares Erzähltalent zu regulieren. Das auf den ersten Blick auffallende wilde Fabulieren hat dementsprechend Methode und zielt auf die wahre Intention des Autoren: 
Seiner Mitwelt die Mißstände vor Augen zu führen und sie zur Umkehr auf die rechte Lebensweise zu bringen.

Es zeugt von dem Wert und der Größe des Werkes, daß diese Botschaft auch heute noch, mehr als 300 Jahre nach der Erscheinung des Buches, kaum etwas an Aussagekraft verloren hat. Mit dem Simplicissimus gab Hans Jakob Christoph von Grimmelshausen der deutschen Literatur ein Werk, das heute zu Recht zu den Klassikern der Weltliteratur gezählt wird. 


\title{
QUELLENNACHWEIS
}

\author{
PRIMÄRLITERATUR
}

Grimmelshausen, Der abenteuerliche Simplicissimus (Darmstadt: Wissenschaftliche Buchgesellschaft, 1985)

---, Simplicianische Schriften (München: Winkler Verlag, 1958)

\section{SEKUNDÄRLITERATUR}

Alewyn, Richard, "Grimmelshausen Probleme," in G. Weydt, Der Simplicissimusdichter und sein Werk (Darmstadt: Wissenschaftliche Buchgesellschaft, 1969): 389-408

Alt, Johannes, "Selbstbiographie und Typenaufbau," in G. Weydt, Der Simplicissimusdichter und sein Werk (Darmstadt: Wissenschaftliche Buchgesellschaft, 1969): 179-205

Battafarano, Italo, "Grimmelshausens Kritik an der Ideologie seiner Zeit," Daphnis 5, 2-4, (1976): 295-302

Breuer, Dieter, "Grimmelshausens politische Argumentation," Daphnis 5, 2-4, (1976): 303-333

Brie, Renate, Die sozialen Ideen Grimmelshausens (Lübeck: Matthiesen Verlag, 1938)

Böckmann, Paul, "Die Abwendung vom Elegantaideal in Grimmelshausens Simplicissimus," in Formengeschichte der deutschen Dichtung Bd.1 (Hamburg: Hoffmann \& Campe, 1967)

Böttcher, Kurt, Lexikon deutscher Schriftsteller (Leipzig: Bibliographisches Institut, 1987) 
Busch, Walter, Hans Jakob Christoffel von Grimmelshausen (Frankfurt/Main: Verlag Moritz Diesterweg, 1988)

Clark, George, The Seventeenth Century (Oxford: University Press, 1969)

Cohn, Egon, Gesellschaftsideal und Gesellschaftsroman des 17. Jahrhunderts (Berlin: Emil Ebering Verlag, 1921)

Daly, Peter, "Simplicissimus Teutsch - doch ein Bildungsroman?" in Alte und Neue Kontroversen, Akten des VII. Internationalen Germanistenkongresses Göttingen, 1985 (Tübingen: Max Niemeyer Verlag, 1986): 159-164

Forster, Leonard, "Neo-Latin Tradition and Vernacular Poetry," in G. Hoffmeister, German Baroque Literature: The European Perspective (New York: Frederick Unger Publ. 1983): 87-108

Friedrich, Carl, The Age of Baroque (New York: Harper \& Row, 1952)

Gaede, Friedrich, Humanismus, Barock, Aufklärung (Bern \& München: Francke Verlag, 1971)

-.-, "Grimmelshausen und der Materialismus" in Alte und Neue Kontroversen, Akten des VII. Internationalen Germanistenkongresses Göttingen, 1985 (Tübingen: Max Niemeyer Verlag, 1986): 165-169

Gebauer, Hans Dieter, Grimmelshausens Bauerndarstellung:

Literarische Sozialkritik und ihr Publikum (Marburg:

N. Elwert Verlag, 1977)

Gebhardt, Bruno, Handbuch der deutschen Geschichte Bd. 2 (Stuttgart: Union Verlag, 1961)

Gundolf, Friedrich, "Grimmelshausen und der Simplicissimus," in Deutsche Vierteljahresschrift, I, (1923): 339-358

Heckmann, Herbert, "Grimmelshausen, oder die Leiden eines Volksschriftstellers," in W. Hinderer, Literarische Profile deutscher Dichter von Grimmelshausen bis Brecht (Königstein: Athenäum Verlag, 1982): 11-24 
Herbst, Gisela, Die Entwicklung des Grimmelshausenbildes in der wissenschaftlichen Literatur (Bonn: Bouvier \& Co., 1957)

Holborn, Hajo, History of Modern Germany 1648 - 1840 (New York: Alfred Knopf, Publ., 1973)

Ingen, Ferdinand von, "Krieg und Frieden bei Grimmelshausen," in Etudes Germaniques, v. 46, 1, (Jan - Mar 1991): 3-20

Joldersma, Hermine \& Wickert, Elke, "Moraldidaktik, Erzählform und Autorintention in Grimmelshausens Landstörzerin Courasche," in The Germanic Review, v. 64, 4 (fall1989): $158-166$

Koschlig, Manfred, Das Ingenium Grimmelshausens und $\geq$ das Kollektiv< (München: C. H. Beck Verlag, 1977)

---, "Grimmelshausens Schriften in den Meßkatalogen 1665 - 1675," in G. Weydt, Der Simplicissimusdichter und sein Werk (Darmstadt: Wissenschaftliche Buchgesellschaft, 1969): 103-11

Könnecke, Gustav, Quellen und Forschungen zur Lebensgeschichte Grimmelshausens (Weimar: Hrsg. J. H. Scholte, 1926)

---, "Der Dichter und sein Held: Hanau - Kroatenlager - Kassel," in G. Weydt, Der Simplicissimusdichter und sein Werk

(Darmstadt: Wissenschaftliche Buchgesellschaft, 1969): 17-55

Lochner, Rudolf, Grimmelshausen, ein deutscher Mensch im 17. Jahrhundert (Reichenberg: Sudetendeutscher Verlag Franz Kraus, 1924)

Lugowski, Clemens, "Literarische Formen und lebendiger Gehalt im Simplicissimus," in G. Weydt, Der Simplicissimusdichter und sein Werk (Darmstadt: Wissenschaftliche Buchgesellschaft, 1969): 161-178

Malettke, Klaus, "Die politische Organisation des Reiches zur Zeit des Simplicissimus," in Etudes Germaniques, v. 64, 1, (Jan Mar 1991): 3-20 
Mannack, Eberhard, "Grimmelshausens Rist - Lektüre und die Folgen," in Chloe, Beihefte zum Daphnis Bd. 3 (Amsterdam: Rodopi, Hrsg. M. Bircher, J. U. Fechner, G. Hillen, 1984): 279-294

Martino, Alberto, "Barockpoesie, Publikum und Verbürgerlichung der literarischen Intelligenz," in Internationales Archiv für Sozialgeschichte der deutschen Literatur, 1, (1976): 107-145

Meid, Volker, Grimmelshausen. Epoche - Werk - Wirkung (München: Verlag C. H. Beck, 1984)

Petersen, Julius, "Hans Jacob Christoph von Grimmelshausen," in G. Weydt, Der Simplicissimusdichter und sein Werk (Darmstadt: Wissenschaftliche Buchgesellschaft, 1969): 56-81

Schaeffer, Peter, "Baroque Philology: The Position of German in The European Family of Languages," in G. Hoffmeister, German Baroque Literature: The European Perspective (New York: Frederick Unger, Publ., 1983): 72-84

Singer, Herbert, Der galante Roman (Stuttgart: Metzlersche Verlagsbuchhandlung, 1961)

Schmiedt, Franz Roland, Deutsche Geschichte Bd. 1 (Berlin: Deutscher Verlag der Wissenschaften, 1967)

Scholte, Jan Henrik, Der Simplicissimus und sein Dichter (Tübingen: Max Niemeyer Verlag, 1950)

Stark, Ernst Eugen, "Barockprosa im Deutschunterricht," in Jentzsch, Brauneck, Stark, Das 17. Jahrhundert in neuer Sicht (Stuttgart:

Ernst Klett Verlag, 1969): 46-92

Stark, Gary, "Vom Nutzen und Nachteil der Literatur für die Geschichtswissenschaft," in German Quarterly (winter 1991): $19-30$

Stern, Martin, "Geld und Geist bei Grimmelshausen," in Daphnis 5, 2-4 (1976): 415-465

Stoll, Christoph, Hans Jacob Christoffel von Grimmelshausen (München: Heinz Moos Verlag, 1976) 
Streller, Siegfried, Grimmelshausens simplicianische Schriften. Allegorie, Zahl und Wirklichkeitsdarstellung (Berlin:

Rüttgen \& Loening, 1957)

Teuscher, Gerhard, "Fromme tugendhafte Frauen oder arglistiges Weibervolk?" in Jahrbuch für Internationale Germanistik, 16, 1 (1984): 94-115

Weydt, Günther, "Grimmelshausens Bildung," in Alte und Neue Kontroversen, Akten des VII. Internationalen Germanistenkongresses Göttingen, 1985 (Tübingen: Max Niemeyer Verlag, 1986): $170-175$

---, Hans Jacob Christoph von Grimmelshausen (Stuttgart: Metzlersche Verlagsbuchhandlung, 1971)

---, Nachahmung und Schöpfung im Barock (Berlin: Francke Verlag, 1968) 